\title{
Ionic Dipolar Switching Hinders Charge Collection in Perovskite Solar Cells with Normal and Inverted Hysteresis
}

Osbel Almora ${ }^{\mathrm{a}, \mathrm{b}, \mathrm{c}, *}$, Pilar Lopez-Varo ${ }^{\mathrm{d}}$, Kyung Taek Cho ${ }^{\mathrm{e}}$, Sadig Aghazada ${ }^{\mathrm{e}}$, Wei Meng, Yi Hou ${ }^{\mathrm{a}}$, Carlos Echeverría-Arrondo ${ }^{\mathrm{b}}$, Iwan Zimmermann ${ }^{\mathrm{e}}$, Gebhard J. Matt ${ }^{\mathrm{a}}$, Juan A. Jiménez-Tejada ${ }^{\mathrm{d}}$, Christoph J. Brabec ${ }^{\mathrm{a}}$, Mohammad Khaja Nazeeruddin ${ }^{\mathrm{e}}$, Germà Garcia-Belmonte ${ }^{\mathrm{b}, *}$

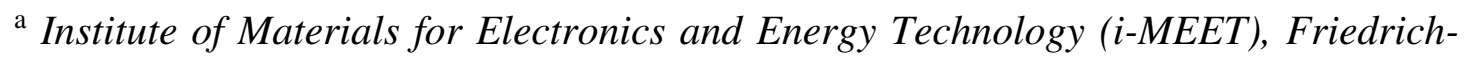
Alexander Universität Erlangen-Nürnberg, 91058 Erlangen, Germany

${ }^{\mathrm{b}}$ Institute of Advanced Materials (INAM), Universitat Jaume I, 12006 Castelló, Spain

c Erlangen Graduate School in Advanced Optical Technologies (SAOT), FriedrichAlexander Universität Erlangen-Nürnberg, 91052 Erlangen, Germany

d Departamento de Electrónica y Tecnología de Computadores CITIC-UGR, Universidad de Granada, 18071 Granada, Spain

e Group for Molecular Engineering of Functional Materials, Institute of Chemical Sciences and Engineering, École Polytechnique Fédérale de Lausanne, CH-1951 Sion, Switzerland

* corresponding authors

E-mail addresses: osbel.almora@fau.de (O. Almora), garciag@uji.es (G. GarciaBelmonte)

Keywords: perovskite solar cells, ionic polarization, hysteresis, photocurrent 


\section{Abstract}

Drift-diffusion modeling of the ionic dipole switching from the measurement of fast scanned and long pre-biased electrical response is proposed as a novel protocol for evaluation of limit hysteretic effects in perovskite solar cells. Up to eight systems were measured including $\quad \mathrm{CH}_{3} \mathrm{NH}_{3} \mathrm{PbI}_{3}, \quad \mathrm{Cs}_{0.1} \mathrm{FA}_{0.74} \mathrm{MA}_{0.13} \mathrm{PbI}_{2.48} \mathrm{Br}_{0.39} \quad$ and $\mathrm{FA}_{0.83} \mathrm{MA}_{0.17} \mathrm{~Pb}_{1.1} \mathrm{Br}_{0.22} \mathrm{I}_{2.98}$ 3D perovskite absorbers, as well as $2 \mathrm{D}$ capping layers towards the selective contacts. We show systematic hysteretic patterns, even among typical hysteresis-free devices, including normal and inverted hysteresis as general dissimilar trend between $\mathrm{CH}_{3} \mathrm{NH}_{3} \mathrm{PbI}_{3}$ and mixed perovskite cells, respectively. Particularly, strong changes in the short-circuit current density $\left(J_{s c}\right)$ were identified, in addition to different trends affecting the fill factor (FF) and the open-circuit voltage ( $V_{o c}$ ). The changes in $J_{s c}$ were analyzed with state-of-the-art numerical drift-diffusion simulations concluding in an important reduction in the charge collection due to ionic distribution switching depending on the pre-biasing protocol and the type of absorbing perovskite. It is shown that mixed perovskites inhibit ionic dipolar switching. In addition, our calculi signal on the required conditions for the occurrence of inverted hysteresis and changes in the $V_{o c}$. Regarding the FF and $V_{o c}$ patterns a new empirical approach is introduced and corresponding interpretations are proposed. 


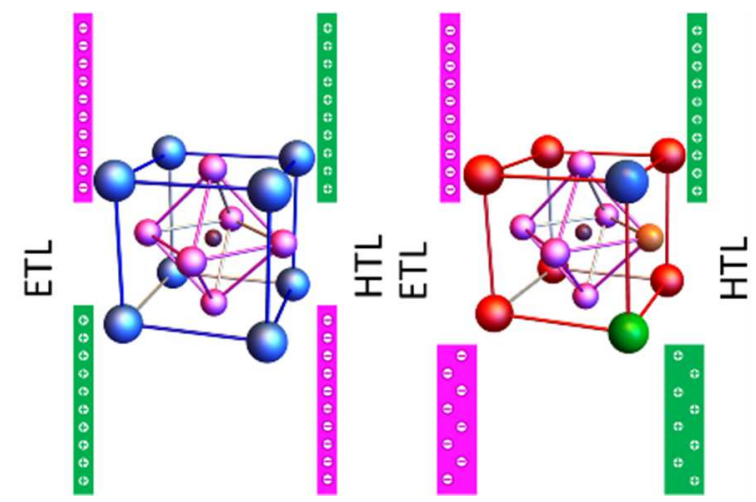




\section{Introduction}

Triggering in 2012 with the introduction of $\mathrm{CH}_{3} \mathrm{NH}_{3} \mathrm{PbI}_{3}$ (MAPI) as absorber material, perovskite solar cells (PSCs) peaked unprecedented increase rates of power conversion efficiency (PCE), recently reaching values above $23 \%$ with facile solution processing. Obviously, an extraordinary growth of related publications appeared (see Fig. S1) [1-3], being the current main research task focused on fabricating high efficiency PSCs exhibiting long-term stability (40-60\% of publications, see Fig. S1b,c). However, apart from the obvious challenge associated to the photovoltaic material science, the device physics of PSCs has been particularly puzzling due to a set of anomalous behaviors which prevent the application of standard characterization protocols $[4,5]$. This is the case of the hysteresis in current density-voltage $(J-V)$ curves -basically the $J-V$ curve in PSCs is critically dependent on the way you measure it- which can be described as the effect of several dynamic charge modulating processes which inhibit steady-state current response in typical time scales. This subject occupies around 20-30\% of publications related to PSCs (see Fig. S1b,c) and the nature of these processes has been widely tackled in the literature [5-10] being the hypothesis of ionic-electronic phenomena the most accepted one at this point.

The $J-V$ characteristic of PSCs, and so the hysteresis, is highly sensitive to several experimental parameters such as the bias sweep direction, scan rate, light intensity and polarization history [5, 8-12]. Frequently, when analyzing this, experimentally as well as using numerical modeling, wide ranges of several variables must be verified with significantly time consuming protocols in order to get some phenomenology feedback 
from systematic trends $[4,5,9]$. As result, most models reproduce a particular hysteretic pattern or family of patterns (e.g. see Fig. S3) [5, 11, 13-16], often exhibiting low repeatability in other similar devices and/or conditions. In order to avoid such problematic situations two main considerations should be taken, in what we call ionic dipoles switching measurement protocol. First, as previously pointed out in our work [17], the faster possible scan rate should be used, when measuring the $J-V$ curves, in order to neglect capacitive displacement currents $J_{c a p}$ and/or non-capacitive contributions $J_{\text {non-cap }}$, such as reactivity (see Fig. S4a). Secondly, a prior polarization (aka stabilization potential) should be applied before each voltage sweep during a prudent time $t_{\text {pre- }}$ larger than the approximate decay of photo-current and photo-voltage (see Fig. S4b). As a result, the analyzed curves may allow differentiating limit effects among mechanisms occurring in two time scales: slower or faster than the sweep time (voltage window over scan rate).

In this work, we present a drift-diffusion-based theoretical analysis on the hysteresis of $J-V$ curves measured at fast scan rates after significant pre-bias from several PSCs, i.e. our ionic dipoles switching experiment. Specifically, the effects of 2D capping layers at the interfaces of devices including $\mathrm{CH}_{3} \mathrm{NH}_{3} \mathrm{PbI}_{3}$ (MAPI) and $\mathrm{Cs}_{0.1} \mathrm{FA}_{0.74} \mathrm{MA}_{0.13} \mathrm{PbI}_{2.48} \mathrm{Br}_{0.39}$ (CFMPIB) are checked and correlated with different types of ionic dipolar profiles. Also typical hysteresis-free devices with organic selective contacts including $\mathrm{FA}_{0.83} \mathrm{MA}_{0.17} \mathrm{~Pb}_{1.1} \mathrm{Br}_{0.22} \mathrm{I}_{2.98}$ (FMPBI) as absorber were measured and shown to present significant inverted hysteresis (IHyst) as mixed perovskite absorber devices. A reduction in the $J_{s c}$ in the form of normal hysteresis (NHyst) after negative 
pre-bias is experimentally reported and confirmed in our simulations for MAPI-cells as a general trend. Negative pre-biasing hinders charge collection at the electrodes and, simultaneously, triggers charge recombination both in the absorber bulk and outer interfaces. These current features respond to the bias-induced inversion of ionic dipole layers. On the contrary, mixed-perovskites structures exhibit short-circuit current enhancement and inverted hysteresis after negative pre-biasing. It is first time revealed here that this is related to the inhibition of the dipolar switching.

Our results validate the proposed experimental methodology for the characterization of the hysteresis in PSCs, joining less time consuming and more reproducible experimental practices with a formal theoretical approach. We also signal the occurrence of at least two main types of re-ordered ionic charge profiles for the hysteretic mechanisms in parallel configuration. One of these is bias-independent for the measurement conditions, and it seems to be exclusively related with the interphase phenomena, recombination and the ionic dipolar switching. The second one could be associated with structural specificities as low crystallinity/grain boundaries/pin holes through which charge transport channels in the bulk can shunt the operating currents. In this sense an empirical approach is introduced.

\section{Experimental}

The fabrication of the devices is described in our previous works [18-20] and, in addition, a summary is provided in the Supporting Information (see section S2 and Fig. S5), also with the specifications for the FMPBI cells. The labeling of the different studied structures is in Fig. 1. All electrical characterizations were carried out at room 
conditions (humidity bellow 10\%). For the electrical measurements two potentiostats were used: SP-200 BioLogic and PP241 Zahner. For the illuminated experiments an AAA solar simulator from Newport equipped with a 1000 W Xenon lamp was employed. The illuminated $J-V$ characteristics before the characterization routine are presented in Fig. S5 and the corresponding data are summarized in Table S1, in which the performance parameters, open-circuit voltage $\left(V_{o c}\right)$, short-circuit current $\left(J_{s c}\right)$, fill factor (FF) and PCE, can be found. Some of these data are reproduced from our previous work [18], since the characterization presented here was followed by an IS analysis (see ref. [18]) before the stressed PCE evaluation. All the data regarding $\mathrm{FA}_{0.83} \mathrm{MA}_{0.17} \mathrm{~Pb}_{1.1} \mathrm{Br}_{0.22} \mathrm{I}_{2.98}$ samples was exclusively measured for this study.

The theoretical description of the mix compound $\mathrm{Cs}_{0.13} \mathrm{FA}_{0.74} \mathrm{MA}_{0.13} \mathrm{PbI}_{2.48} \mathrm{Br}_{0.39}$ is based on density functional theory (DFT) calculations carried out with VASP; a code implementing the projector augmented-wave method (PAW), on a unit cell containing 1096 atoms. Computations are run at the gamma point using a plane-wave cutoff energy of $450 \mathrm{eV}$ and PAW pseudopotentials generated in the generalized gradient approximation of Perdew-Becke-Ernzerhof to the exchange-correlation functional. The atomic positions in the supercells are relaxed until forces acting on individual nuclei become smaller than $0.1 \mathrm{eV} \cdot \AA^{-1}$. The dielectric constants of the mix compounds (Fig. 2b) are obtained in the independent-particle approximation.

\section{Results and Discussion}

\subsection{Experimental J-V curves from ionic dipolar switching protocol.}


To facilitate our discussion, we first clarify some notations and considerations. Here we call forward/positive (F) bias to an applied voltage between short-circuit (SC) and opencircuit (OC) conditions, and reverse/negative (R) bias to that bias voltage below SC, as depicted in the sketch of Fig. S2. Therefore, the voltage can be swept from SC to OC (RF) or from OC to SC (FR) [3, 5, 13]. Accordingly, hysteretic features are usually checked by sweeping the voltage in RF and FR directions and the resulting currents, $J_{R F}$ and $J_{F R}$ respectively, are then compared while systematically changing a given parameter. The subsequent evaluation of the current difference

$$
\Delta J=J_{F R}-J_{R F}
$$

is firstly a source of uncertainties regarding the report of the PCE: there will be a different efficiency for each set of measurement parameters. Secondly, the hysteresis informs on the dynamic evolution of charge due to slower mechanisms (from milliseconds to minutes) overlapping and modifying the device operating currents

$$
J_{\text {oper }}(V)=J_{s}\left(\exp \left[\frac{q\left(V-R_{\text {series }} J\right)}{m k_{B} T}\right]-1\right)+\frac{\left(V-R_{\text {series }} J\right)}{R_{\text {sh }}}-J_{p h}
$$

where $J_{p h}$ is the photocurrent, $J_{s}$ is the diode saturation current, $R_{s h}$ the shunt resistance, $q$ the elementary charge, $m$ is the ideality factor, $k_{B} T$ the thermal energy, $V$ the applied voltage and $R_{\text {series }}$ the series resistance.

Keeping this in mind, we can take a look to the $J-V$ curves presented in Fig. 1b,d, where the applied voltage sweep is as fast as $5.0 \mathrm{~V} \cdot \mathrm{s}^{-1}$ after 1 minute stabilization at the edges of the voltage window, i.e. $-0.1 \mathrm{~V}$ and $1.2 \mathrm{~V}$. Illustrative of the current-time evolution during pre-bias are the curves in Fig. S6a-b. In this experiment, only processes 
that occur in less than $200 \mathrm{~ms}$ will be detected in each scan, thus the effect of the slowest processes will "freeze". Accordingly, the measurement can be considered as a pseudo-steady state whose boundary conditions are defined during the pre-bias period. Using other measurement conditions (slower sweeps and/or different pre-biasing) can generate diverse hysteretic patterns by the overlapping of different processes, including reactivity (see examples of Fig. S6c).

In Fig. 1b, an abrupt decrease of photocurrent $\Delta J_{s c}$ after reverse pre-biasing in the RF sweep is observed as the main feature for MAPI-cells with 2D capping. This decrease is larger on samples with $\mathrm{Bl}_{2} \mathrm{PbI}_{4} /$ spiro-OMeTAD interphase. In addition, a significant reduction of the FF occurs in the RF scan with respect to the FR sweep. This latter pattern seems to occur independently of the presence of the $2 \mathrm{D}$ capping layers.

Note from Eq. (1) that $\Delta J$ (and thus $\Delta J_{s c}>0$ ) is positive for all the MAPI cells in the range between SC and OC, usually labeled as normal hysteresis (NHyst). Thus two more cases can occur: $\Delta J<0$ or $\Delta J \approx 0$, when inverted hysteresis (IHyst) or negligible hysteresis, respectively $[5,21]$. 
(a)

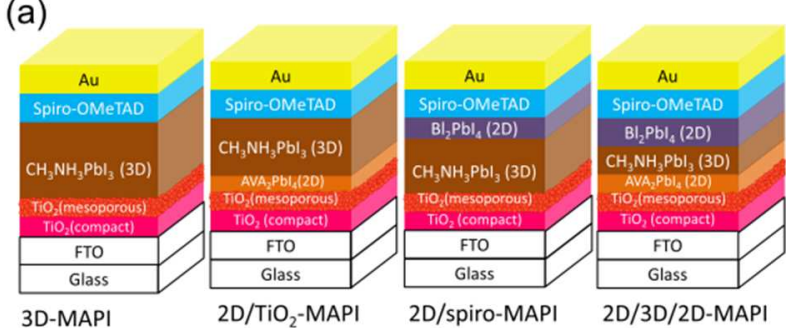

(b)

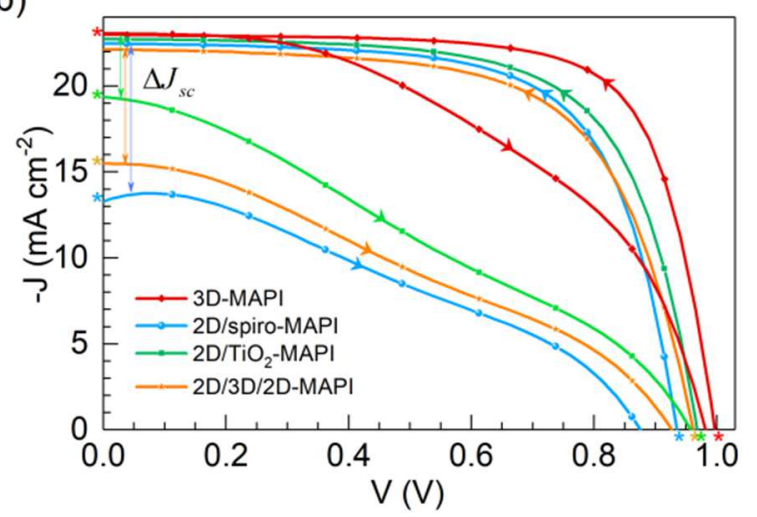

(c)

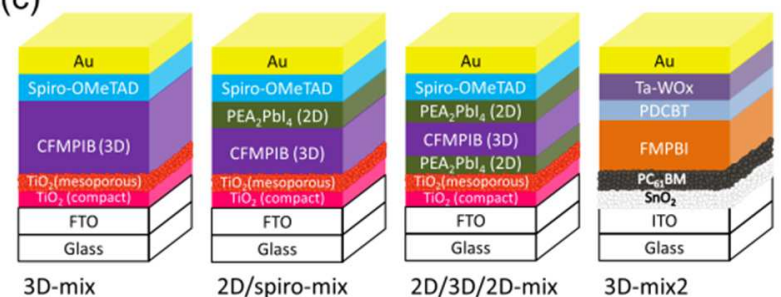

(d)

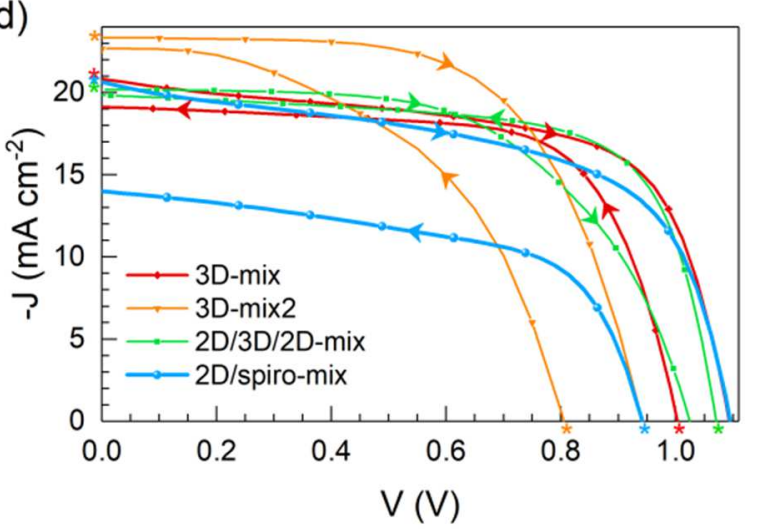

Fig. 1. MAPI-based devices, schemes of structures in (a) and experimental $J-V$ curves in (b), show NHyst with evident changes of $J_{s c}$ and FF depending on the 2D capping layers and the scan directions, respectively. Mixed perovskite cells, schemes in (c) and curves in (d), show IHyst with a main shift on the 
$V_{o c}$. These dissimilar trends are suggested by our drift-diffusion simulations to be due to differences between MAPI and the mixed perovskites, e.g. $\mu$ and $\varepsilon$. The $J-V$ curves were measured at $5 \mathrm{~V} \mathrm{~s}^{-1}$ under 1 sun standard illumination after 1 minute pre-biasing at $-0.1 \mathrm{~V}$ and $1.2 \mathrm{~V}$ for RF and FR scans, respectively. Scaling between layers thicknesses was not considered in the schemes. The arrows and stars in the $J-V$ curves signal the sweep directions and pre-bias, respectively.

The experimental $J-V$ curves for the mixed perovskite samples are illustrated in Fig. 1d. The value of $\Delta J_{s c}$ is significant for the device including 2D capping on the spiroOMeTAD contacting layer, with the specificity that now the current trends are reversed resulting in IHyst (negative $\Delta J_{s c}$ ). In addition, the effect of the $1.2 \mathrm{~V}$ pre-bias reduces the $V_{o c}$ beyond the expected logarithmic drop corresponding to $\Delta J_{s c}$. Interestingly, this $V_{o c}$ reduction is quite evident in the 3D-mix2 (FMPBI-based), which responds to a structure typically assumed to be hysteresis-free (see Fig. S5d).

Importantly, let us highlight some differences between the devices including MAPI and CFMPIB as absorber materials, apart from showing NHyst and IHyst, respectively. From our previous work [18], we have found that (i) Shockley-Read-Hall recombination due to mid-gap states could be the predominant mechanism in MAPI ( $m \approx 2)$ while for CFMPIB the influence of bulk or even band-to-band recombination cannot be ignored ( $m \approx 1.5$ ), the later considering $V_{o c}$ values close to the radiative limit for the most optimized devices [4]. Also (ii) the field screening is expected to behave differently since the relative permittivity $\varepsilon$ is larger for MAPI than for mixed absorbers. The latter 
is observed experimentally (Fig. 2a) as well as theoretically: while the literature reports $\varepsilon>25$ for MAPI [22-25], we found $\varepsilon=15.7$ for CFMPIB from our Density Functional Theory (DFT) calculations (Fig. 2b). Furthermore, (iii) cells with CFMPIB presented inductive element behavior in the IS spectra as the illumination intensity approached and exceeded 1 sun [18].
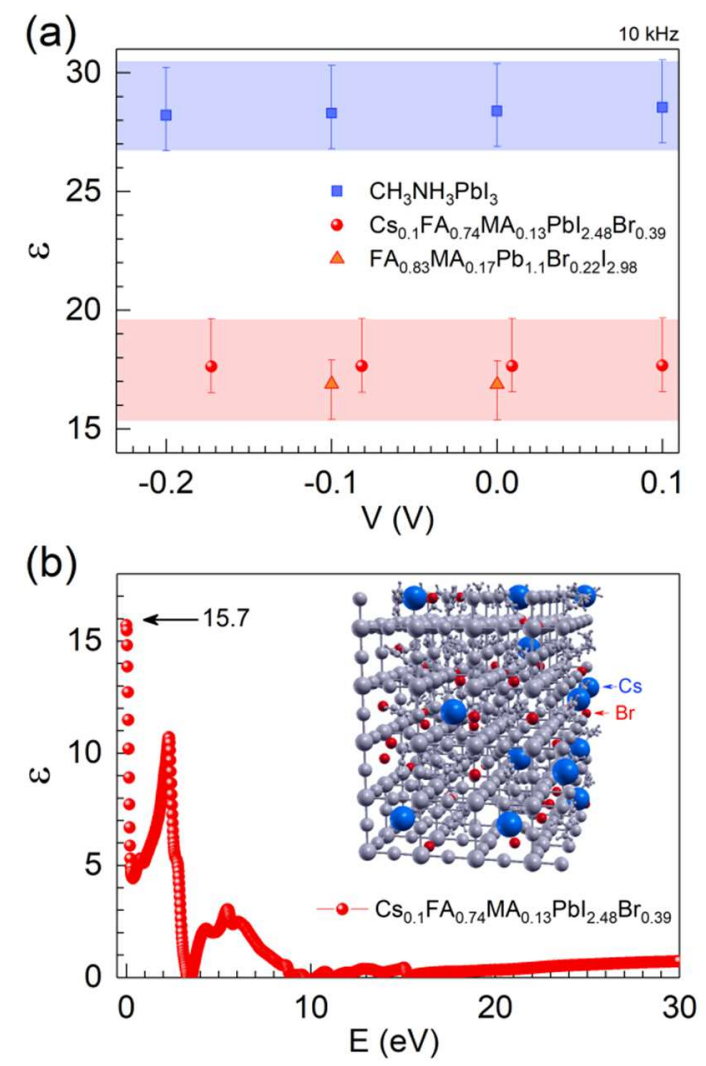

Fig. 2. (a) Experimental dielectric constant as a function of reverse bias of 3D-MAPI, 3D-mix and 3Dmix 2 samples for absorber thickness around $200 \mathrm{~nm}, 300 \mathrm{~nm}$ and $600 \mathrm{~nm}$, respectively, as indicated with error bars. From our simulations, a lesser $\mathcal{E}$ is one among four conditions in order to obtain ionic dipolar switchable phenomena behind inverted hysteresis. The measurements of geometrical capacitance were made at $10 \mathrm{kHz}$, dark, room conditions (see ref. [18]). (b) Theoretical estimation of dielectric constant for CFMPIB from DFT calculations. Inset the used array of atoms highlighting the random distribution of inorganic atoms differing from MAPI. 


\subsection{Charge collection and the change in $J_{s c}$ by drift-diffusion simulation.}

From the above results, $\Delta J_{s c}$ is found to signal distinctive trends: it behaves by way of NHyst for the MAPI-cells and as IHyst in the mixed-perovskite-samples. Particularly, after the negative pre-biasing, $J_{s c}$ is observed to be reduced (MAPI-cells) or enlarged (mixed-perovskite cells), which informs on diverse interfacial ionic accumulation patterns depending of the solar cell structure.

In order to interpret the $\Delta J_{s c}$ experimental results, two $J-V$ curves have been simulated for an ETL/MAPI/HTL cell (electron/hole transporting layers sandwiching the perovskite) with 2D interfacial dipole layer effects (see Fig. 3a and the simulation parameters of Table S2). The $J-V$ curves of Fig. 3a correspond to a fast FR scan and a fast RF scan, reproducing the experimental curves reported in Fig. 1b, which we correlate with ionic dipolar switching. Intermediate values with respect to the $\Delta J_{s c}$ in Fig. 3a can be easily simulated by slightly modifying parameters in Table S2. Four points of the scans of Fig. 3a ("A" and "D" in SC, and "B" and "C" at OC) have been selected to calculate the energy diagrams and other electrical variables along the structure. Importantly, note that the "ionic dipolar switching" refers to (i) not just ions that pile up, but ions that accumulate in specific configurations for each electrical response, that (ii) are switched drastically (inverting ionic dipole layer sign) during the proposed experiment (pre-bias inversion) but dynamically when typical curve measurements are performed. 

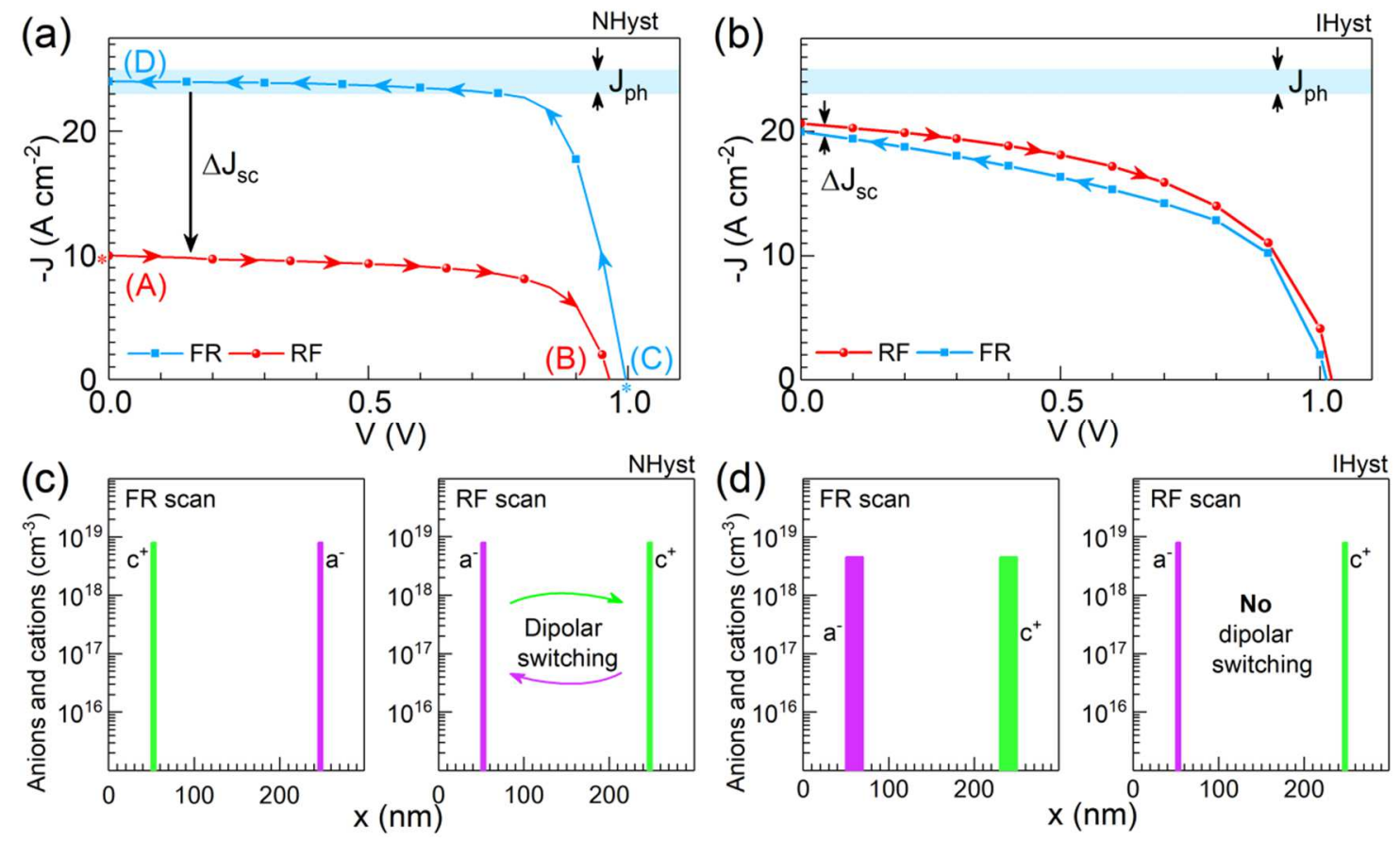

Fig. 3. Simulated $J-V$ curves indicating (a) NHyst and (b) IHyst as result from the ionic profiles of (c) and (d), respectively. Different absorber materials (MAPI or mixed perovskite) enables different ionic dipolar distribution which correlates simulated patterns with experimental curves in Fig. 1b,d. In (a) we highlight the points in which energy band diagrams are presented through the work. The calculations were made with the parameters in Table S2. The arrows indicate the sweep directions and the light blue sections highlight the approximate photo-current $\left(\sim 24 \mathrm{~mA} \mathrm{~cm}^{-2}\right)$. Mixed NHyst and IHyst is also shown in Fig. S7. The following assumptions were considered: (i) the scan is fast enough to consider that ions do not migrate while scanning, and (ii) the initial distributions of ions are previously calculated or defined.

The numerical model used in this work $[5,26,27]$ was initially proposed for an ETL/perovskite/HTL structure and now adapted to include the 2D interfacial dipole layers effects. The core of the numerical modeling includes the following set of differential equations: (1) drift-diffusion equations, (2) Poisson's equation, and (3) continuity equations for electron and holes. As we consider fixed distribution of ions 
during the fast scans, they are modelled as a dipolar distribution located in sheets of charge at the interfaces (see Fig. 3c, in which the distribution of ions for the FR and RF scans used for Fig. 3a, are represented) [26].

Our model also includes different physical mechanisms which can help to interpret the effect of the 2D layers (see also Fig. S8): (i) a discontinuity in the conduction band energy, $\Delta E_{C}$, at the ETL/perovskite interface, and a discontinuity in the valence band energy, $\Delta E_{V}$, at the HTL/perovskite interface; (ii) surface recombination rate, $R_{S}$, between electrons and holes accumulated at the interfaces; (iii) bulk recombination rate, $R_{B}$, in the perovskite, and (iv) migration of ions. The energy diagrams calculated at points " $A$ " and "D" in SC for the fast scans of Fig. 3a are represented in Fig. 4a,b (the energy diagrams of the points "B" and "C" of Fig. 3a are in Fig. S9a,b). Further corresponding electrical variables are presented in Fig. S10 and Fig. S11.

Fig. 4a shows our simulated band diagrams at $\mathrm{SC}$ in the fast RF scan starting after $1 \mathrm{~min}$ pre-biasing at $-0.1 \mathrm{~V}$. This is actually a statement of good practices since we avoid typical qualitatively sketched energy diagrams based on notional considerations $[15,16$, 28-30]. The band levels are bended by the dipolar distribution formed by anions (purple circled minuses) in front of the ETL and cations (green circled pluses) just in front of the HTL, creating barriers for electrons and holes after the negative pre-biasing. This situation is reversed in SC in Fig. 4b, in a sort of switching after the positive pre-biasing, where there is no major obstacle for the charge collection. Accordingly, significantly larger recombination rates are obtained after the $-0.1 \mathrm{~V}$ pre-bias because of the reduced charge collection, both at the interfaces and in the bulk, being the $R_{B}$ difference lightly shifted towards the ETL contact, in Fig. 4c. Therefore, drastic changes are expected in the electronic and ionic distributions, potential, electric field and current density, as 
shown in Fig. S10. All these changes in the electrical variables are the origin of the large variation of $\Delta J_{s c}$ observed in Fig. 3a, which hinders charge collection at the electrodes and, simultaneously, triggers charge recombination both in the absorber bulk and outer interfaces. This is fostered by the inversion of the ionic dipole layers after poling.

Importantly, a higher discontinuity in the valence band energy at the HTL/perovskite interface increases the value of the electronic accumulated charge at this interface [27]. In addition, a higher value of surface recombination constant, $R_{s}$, can modify this accumulation of charge and the magnitude of the current at SC [31]. Thus, in SC, modifications at the perovskite/HTL interface can produce significant variations of $J_{s c}$.

A parallel analysis for the OC condition can be seen in Fig. S9 (energy diagrams) and Fig. S11 (distribution of electrical variables). Interestingly, despite the significant changes of recombination at SC (Fig. 4c) and the inverted potential and field profiles at OC (Fig. S10c,d), the $V_{o c}$ is nearly the same due to the recombination $R_{B}$ constancy at OC. Also note that the loosing of $J_{s c}$ in MAPI with NHyst (Fig. 1b and Fig. 3a), attributed here to the dipolar switching, requires the presence of the $2 \mathrm{D}$ layers. This could be supported by the reported lower mobility [32-34] which may contribute to form "cages" for the ions during fast scan after the pre-bias. Actually, the statement of suppressed ion migration in $2 \mathrm{D} \mathrm{PEA}_{2} \mathrm{PbI}_{4}$ has been previously suggested from electrothermal characterizations [35]. Some other drift-diffusion simulations [33] have found the charge recombination rate constants to be one order of magnitude larger for 2D perovskites than in the $3 \mathrm{D}$ parent compound. In addition, note that the interface towards the HTL with the 2D capping favors the accumulation of either cations or anions, depending on the poling sign. 

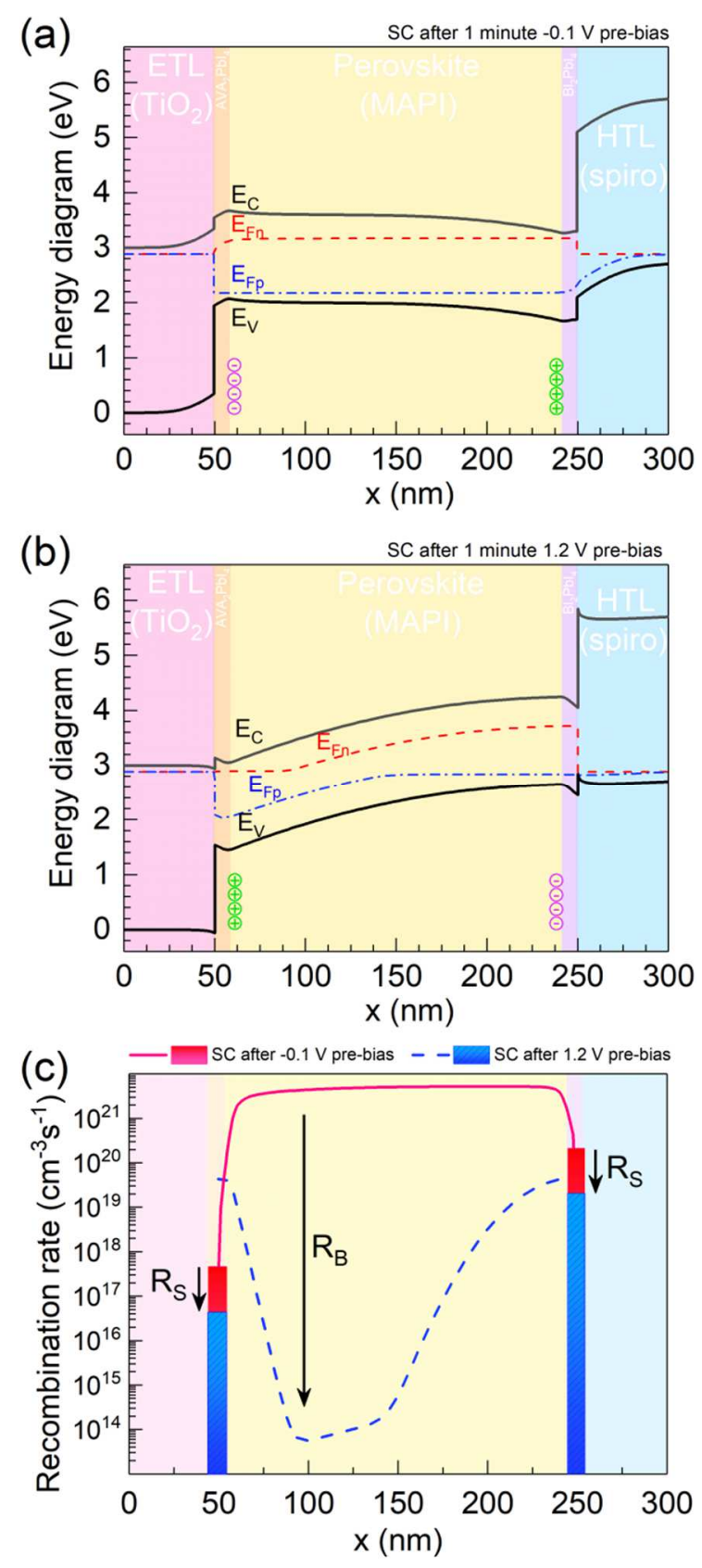

Fig. 4. Simulated energy diagrams of a PSCs including 2D layers working under illumination in shortcircuit (applied $V=0 V$ ) calculated at (a) point "A" in the RF scan and after ionic dipoles switching at (b) point "D" in the FR scan of Fig. 3a. (c) Magnitude of the surface, $R_{\mathrm{S}}$, and bulk, $R_{\mathrm{B}}$, recombination rates for cases (a) and (b). $E_{F n}$ and $E_{F p}$ are the quasi-Fermi levels of electrons and holes, respectively, and $E_{C}$ and $E_{V}$ are the conduction and valence band levels, respectively. Green (+) and purple (-) 
circles symbol cations and anions, respectively. Fitting parameters are in Table S2. The distributions of other electrical variables are represented in Fig. S10.

Numerical NHyst $J-V$ features were reproduced in most of the analysed cases after the testing of large ranges of values for the parameters listed in Table S2. This corroborates the experimental observations of NHyst predominance [5, 13]. As observed in the experimental results, the main difference between MAPI structures (Fig. 1b) and the mixed-perovskite samples (Fig. 1d) is the hysteresis trend: NHyst or IHyst, respectively. IHyst is not frequently observed in PSCs [5, 13], and previous studies propose that its origin can be related to ETL/perovskite interface properties [21], or to pre-conditioning at a certain biasing point [36],

Our numerical simulations show that the existence of IHyst depends on the necessary combination of different factors: (i) a specific initial ion distribution formed at a prebiasing point (as in ref. [36]); (ii) specific values of the surface recombination at selective contacts/perovskite interfaces (in agreement with authors that associate the IHyst with the interface properties) [21]; (iii) an appropriate range of values for the mobility of the carriers in the perovskite semiconductor and (iv) an appropriate range of values for the perovskite relative permittivity. These four factors are needed but not individually sufficient for IHyst; from our simulations only in conjunction IHyst occurs.

Regarding the specific distributions of ions in the perovskite, only when a negative net ionic charge accumulates at the ETL/perovskite interface in steady OC (or 1 min $1.2 \mathrm{~V}$ ) and positive net ionic charge accumulates at the HTL/perovskite interface in steady SC (or $1 \min -0.1 \mathrm{~V}$ ), the $J-V$ curves of a PSC show IHyst. This means that the ionic dipoles are modified by pre-bias, but not switched, which only occurs with mixed perovskites. Furthermore, the IHyst is only reproduced in our simulations when the values of the carrier mobility and relative permittivity are necessarily lower than those used to reproduce NHyst curves. On the later, we highlight the concordance of our driftdiffusion simulations with the $\varepsilon$ values from experiments and DFT calculation in Fig. 2. Interestingly, the lower $\varepsilon$ assumption could be thought to conflict with some previous reports $[14,17,37]$ on IHyst in MAPI-based cells. However, note that these 
reports include no faster or long enough pre-biasing conditions, or consecutive RF-FR scans, which discard the application of our theoretical approach. In our experience none MAPI-based cell reproduced IHyst under our ionic dipolar switching experimental procedure, and if that would occur, the presence of faradaic currents or more complex phenomena not included in the drift-diffusion simulations should be considered.

A simulated IHyst curve is depicted in Fig. 3b. The values of the parameters used in our simulation to reproduce IHyst curves are shown in Table S2. The distribution of ions for the FR and RF scans are represented in Fig. 3d. If we compare the distributions of ions used in the NHyst case (Fig. 3c) and the IHyst case (Fig. 3d), the former shows an inversion of the dipolar ionic distribution when changing from the RF to the FR scans. However, in the IHyst case there is not such a dipolar switching. Instead, for the IHyst case, there is an enlargement of the ionic dipoles after $-0.1 \mathrm{~V}$ pre-bias with respect to the profile after $1.2 \mathrm{~V}$ pre-bias. Also note that the IHyst -or inhibited dipolar switchingdoes not depend on the presence of 2D perovskite capping layers. Additionally, whilst the HTL/2D capping interface favors the ions accumulation, similarly to the MAPI case, a sort of cation-affinity occurs with the spiro which prevents the accumulation of anions towards the HTL, opposite to the MAPI case. This also possibly speaks about the more diverse nature of the mobile ions in the mixed perovskite.

Apart from the pure NHyst and IHyst $J-V$ curves obtained experimentally in Fig. 1b and Fig. 1d, mixed NHyst and IHyst is also observed in our experiments (case $2 \mathrm{D} / \mathrm{TiO}_{2}$ mix in Fig. 1d). There is a crossing between the FR and the RF scans, showing both NHyst and IHyst. This crossing can be attributed to a change in the value of the $V_{o c}$ in the FR and RF scans. The magnitude of the $V_{o c}$ can be modified by a surface recombination at the boundaries of a solar cell [31]. From our simulations, the accumulation of charge at the interfaces at OC is different in the RF or the FR scans. Thus, a change in the value of the $V_{o c}$ can occur as in Fig. $3 \mathrm{~b}$ and Fig. S7. On the other hand, further modifications in the $V_{o c}$ could enhance the IHyst effect as result of local interface polarization processes [21]. These mechanisms -not included in our 
simulations- could complement/overlap the dipolar switching inhibition in order to reproduce the most drastic experimental IHyst features, as in Fig. 1d.

Therefore, to this point our simulations reproduce the main experimental trends of Fig. 1b,d (NHyst, IHyst, $\Delta J_{s c}$ ) correlating the type of absorber (MAPI or mixed perovskite) with the corresponding ionic dipolar distributions. In addition, we highlight two other interesting patterns reproduced in Fig. 3. First, as highlighted in blue, our simulations suggest a good approximation to the value of $J_{p h}$ only when taking $J_{s c}$ from the FR scan in NHyst (Fig. 3a). A significant reduction of $J_{s c}$ was found when RF in NHyst (Fig. 3a) and both scans in IHyst in simulations (Fig. 3b,c) as well as in the experiments in Fig. 1. Second, the reduced FF in both RF and FR scans in a sort of apparent reduced $R_{s h}$, reproduced in Fig. 3b,c, matches the experimental behaviour in Fig. 1d. This effect deserves particular attention in a future work since the $R_{\text {sh }}$ dependency on illumination intensity and polarization routine is still an intriguing issue in general photovoltaics [38, 39].

\subsection{Introducing an empirical approach to fill factor changes.}

As tackled in the previous section, the change of $J_{s c}$ was related to the enhancement in recombination due to ionic dipolar switching. Numerical simulations were able to model $\Delta J_{s c}$ for NHyst as well as changes in $V_{o c}$, and also the occurrence criteria (inhibition of ionic dipolar inversion) for IHyst. However, there is also an important modification of the FF in our experimental $J-V$ curves (mainly for MAPI-devices in Fig. 1b) which is also an often reported hysteretic pattern in the literature [5, 9, 13, 37].

Purposely, the difference in hysteretic current $\Delta J$, introduced in Eq. (1), can be expressed in terms of adding individual terms which may correspond to different parallel elements in the corresponding DC equivalent circuit (see Fig. 5). From the experimental $J-V$ curves of Fig. 1b,d, we propose the empirical form 
$\Delta J(V)=\Delta J_{s c}+J_{\alpha} \alpha(V)-J_{\beta} \beta(V)$

with the step-like functions

$$
\begin{aligned}
& \alpha(V)=\left(1+\exp \left[-\frac{q\left(V-V_{\alpha}\right)}{n_{\alpha} k_{B} T}\right]\right)^{-1} \\
& \beta(V)=\left(1+\exp \left[-\frac{q\left(V-V_{\beta}\right)}{n_{\beta} k_{B} T}\right]\right)^{-1}
\end{aligned}
$$

As noted from Eq. (3), $\alpha$ - and $\beta$-terms introduce positive and negative corrections to $\Delta J$, respectively. They are related to the occurrence of NHyst and IHyst depending on the current sign. In the phenomenological equivalent circuit of Fig. 5, the switchers account for the respective Eq. (3) term occurrence. They are just explanatory tools used to illustrate the occurrence/absence of different processes depending on the system and/or the measurement conditions (e.g. scan rate/direction, pre-bias). For instance, in absence of hysteresis all the currents different from $J_{\text {oper }}$ are switched-off, whilst only $\Delta J_{s c}$ should be switched-on if pure ionic dipolar profiles towards the electrodes occur. Also note that, for instance, around the maxim power voltage $V_{m p p}$ a loss of power up to $J_{\alpha} V_{m p p}$ can be expected when NHyst $\left(J_{\beta} V_{m p p}\right.$ when IHyst) if neglected $\Delta J_{s c}$. In any case, whatever the physical meaning these terms are related with, the parallel distribution of current lines in addition to $J_{\text {oper }}$ might suggest (i) mechanisms in the complete bulk and/or (ii) possibly local channels for currents flowing through grain boundaries, pin-holes and/or surface edge effects, resulting in specific hysteretic patterns. 
The experimental values of $\Delta J$ taken from $J-V$ curves of Fig. 1b,d (dots) are shown in Fig. S12a,b, presenting a good agreement with the fittings to empirical Eq. (3) (solid lines) using the parameters of Table S3. Actually, from all our measurements we found that the parameterization in Eq. (3) fits well the experimental $\Delta J$. Therefore, any detailed model behind that hysteretic behavior should approach Eq. (3) under a set of given parameters.

The nature of the terms and parameters in Eq. 3 and the DC equivalent circuit in Fig. 5 is discussed in the attached Section S3.1 were we hypothesize on a more conventional representation (using diodes). Purposely, we highlight the "S" shape of $J_{\alpha} \alpha(V)$ in Eq. $3 \mathrm{~b}$ and Fig. S12. This shape resembles the $J-V$ curves of polycrystalline CdTe solar cells $[40,41]$ where the high injection current of a main junction diode is limited by an opposing Schottky diode. Consequently, we could hypothesize that $\mathrm{p}^{+} \mathrm{np}$ or $\mathrm{n}^{+} \mathrm{pn}$ local charge density profiles (base-overloaded transistors or switchable consecutive ionic dipoles) are oriented during pre-bias and/or voltage scans in sort of channels which produce NHyst or IHyst, depending on the polarization. These ionic-related charge profiles could behave similarly to the dipolar sheets assumed in our drift-diffusion simulations in quasi-steady-state during the fast scan.

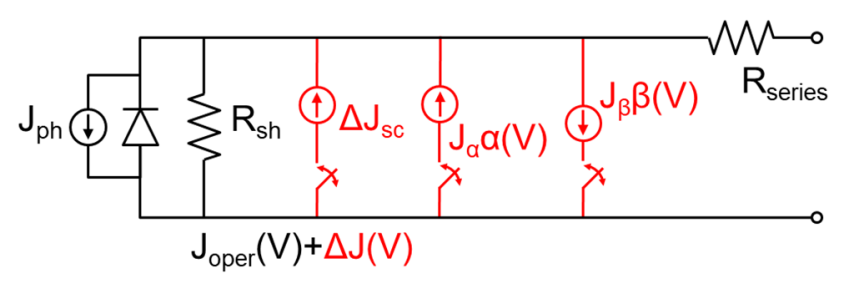

Fig. 5. Equivalent DC circuit corresponding to Eq. 3 where the switch symbols mean the occurrence of 
each current depending on the ionic modulation (scan direction and/or pre-bias). Hysteretic currents are shunted among them and with $J_{\text {oper }}$. Switching off the hysteretic currents may result in the regular operational current.

In addition, we observe that $J_{\alpha} \alpha(V)$ is linked with the occurrence of NHyst (all MAPIdevices and 2D/3D/2D-mix cell), while $J_{\beta} \beta(V)$ predominance seems to be related with the IHyst. The $J_{\alpha} \alpha(V)$ term fades as the IHyst is enhanced. As a result, the hysteresis DC equivalent circuits are represented for NHyst-MAPI-cells and IHyst-CFMPIBdevices in Fig. S12c and Fig. S12d, respectively. Furthermore, from our previous work we found a direct correlation between IHyst and the presence of inductive elements in the equivalent circuit of the impedance spectra [18].

\section{Conclusions}

In summary, our study reinforces the idea that ionic displacement and accumulation at the interfaces lie behind many of the differences usually observed in the measured current of perovskite solar cells and generically labeled as "hysteresis". Our experimental protocol permits disregarding contact reactivity and degradation as the source of the observed changes in performance. Fast $J-V$ scans in combination to 1 min pre-bias allows separately probing electronic and ionic dynamics, and show how ion built-up alters the electronic mechanisms of charge collection and recombination. By drift-diffusion device simulations, experimentally observed (positive or negative) variations in short-circuit current after pre-biasing result from the degree of bias-induced inversion of ionic dipole layers at the interfaces. The reduction in short-circuit current and observation of normal hysteresis is reported for MAPI-cells as a general trend. Negative pre-biasing hinders charge collection at the electrodes and, simultaneously, triggers charge recombination both in the absorber bulk and outer interfaces. On the 
contrary, mixed-perovskites structures exhibit short-circuit current enhancement and inverted hysteresis after pre-biasing. It is revealed here that this is related to the inhibition of the dipolar switching. In addition, a parametric approach possibly illustrating correlation with morphological features was introduced.

\section{Appendix A. Supporting Information}

Supplementary data associated with this article can be found in the online version at http://dx.doi.org/10.1016/j.solmat.2018

\section{Author Information}

Corresponding authors

*E-mail: osbel.almora@fau.de, garciag@uji.es

\section{ORCID}

\begin{tabular}{|c|c|}
\hline Osbel Almora & https://orcid.org/0000-0002-2523-0203 \\
\hline Pilar Lopez-Varo & https://orcid.org/0000-0002-2170-1581 \\
\hline Kyung Taek Cho & https://orcid.org/0000-0002-7419-1415 \\
\hline Sadig Aghazada & https://orcid.org/0000-0002-7568-4481 \\
\hline Wei Meng & https://orcid.org/0000-0002-4887-1732 \\
\hline Xiaofeng Tang & https://orcid.org/0000-0003-1943-4063 \\
\hline Carlos Echeverría-Arrondo & https://orcid.org/0000-0001-6627-5005 \\
\hline Juan A. Jiménez-Tejada & https://orcid.org/0000-0003-2444-3116 \\
\hline Christoph J. Brabec & https://orcid.org/0000-0002-9440-0253 \\
\hline Mohammad Khaja Nazeeruddin & https://orcid.org/0000-0001-5955-4786 \\
\hline Germà Garcia-Belmonte & https://orcid.org/0000-0002-0172-6175 \\
\hline
\end{tabular}

Notes

The authors declare no competing financial interest. 


\section{Acknowledgments}

We acknowledge funding from MINECO of Spain under project MAT2016-76892-C31-R and MAT2016-76892-C3-3-R. O.A. acknowledges Generalitat Valenciana for the grant GRISOLIA/2014/035, the financial support from the VDI/VD Innovation + Technik GmbH (Project-title: PV-ZUM) and the SAOT funded by the German Research Foundation (DFG) in the framework of the German excellence initiative. We thank the Swiss National Funds for Scientific Research contract number 200020L_172929/1, Project "Tailored Design and in-depth understanding of perovskite solar materials using in-house developed 3D/4D nanoscale ion-beam analysis". Borun New Material Technology generously supplied a high-quality spiro-OMeTAD. W.M. and E.G. gratefully acknowledge the financial support from the China Scholarship Council.

\section{References}

[1] J. Bisquert, E.J. Juárez-Pérez, P.V. Kamat, Hybrid Perovskite Solar Cells: the Genesis and Early Developments 2009-2014, Fundació Scito, Valencia, 2017.

[2] M.A. Green, Y. Hishikawa, E.D. Dunlop, D.H. Levi, J. Hohl-Ebinger, A.W.Y. HoBaillie, Solar cell efficiency tables (version 52), Prog. Photovoltaics 26 (2018) 427-436.

[3] O. Almora, L. Vaillant-Roca, G. Garcia-Belmonte, Perovskite Solar Cells: A brief Introduction and some Remarks, Rev. Cubana Fis. 34 (2017) 58-68.

[4] W. Tress, M. Yavari, K. Domanski, P. Yadav, B. Niesen, J.P. Correa Baena, A. Hagfeldt, M. Graetzel, Interpretation and Evolution of Open-Circuit Voltage, Recombination, Ideality Factor and Subgap Defect States during Reversible LightSoaking and Irreversible Degradation of Perovskite Solar Cells, Energy Environ. Sci. (2017).

[5] P. Lopez-Varo, J.A. Jiménez-Tejada, M. García-Rosell, S. Ravishankar, G. GarciaBelmonte, J. Bisquert, O. Almora, Device Physics of Hybrid Perovskite Solar Cells: 
Theory and Experiment, Adv. Energy Mater. 8 (2018) 1702772.

[6] H.J. Snaith, A. Abate, J.M. Ball, G.E. Eperon, T. Leijtens, N.K. Noel, S.D. Stranks, J.T.-W. Wang, K. Wojciechowski, W. Zhang, Anomalous Hysteresis in Perovskite Solar Cells, J. Phys. Chem. Lett. 5 (2014) 1511-1515.

[7] E.L. Unger, E.T. Hoke, C.D. Bailie, W.H. Nguyen, A.R. Bowring, T. Heumuller, M.G. Christoforo, M.D. McGehee, Hysteresis and Transient Behavior in CurrentVoltage Measurements of Hybrid-Perovskite Absorber Solar Cells, Energy Environ. Sci. 7 (2014) 3690-3698.

[8] H.-S. Kim, N.-G. Park, Parameters Affecting I-V Hysteresis of $\mathrm{CH}_{3} \mathrm{NH}_{3} \mathrm{PbI}_{3}$ Perovskite Solar Cells: Effects of Perovskite Crystal Size and Mesoporous $\mathrm{TiO}_{2}$ Layer, J. Phys. Chem. Lett. 5 (2014) 2927-2934.

[9] W. Tress, N. Marinova, T. Moehl, S.M. Zakeeruddin, M.K. Nazeeruddin, M. Gratzel, Understanding the Rate-Dependent J-V Hysteresis, Slow Time Component, and Aging in $\mathrm{CH}_{3} \mathrm{NH}_{3} \mathrm{PbI}_{3}$ Perovskite Solar Cells: The Role of a Compensated Electric Field, Energy Environ. Sci. 8 (2015) 995-1004.

[10] A. Guerrero, A. Bou, O. Almora, T. Heumuller, G. Garcia-Belmonte, J. Bisquert, Y. Hou, C.J. Brabec, Switching Off Hysteresis in Perovskite Solar Cells by Fine-tuning Energy Levels of Extraction Layers, Adv. Energy Mater. 8 (2018) 201703376.

[11] G.A. Nemnes, C. Besleaga, A.G. Tomulescu, I. Pintilie, L. Pintilie, K. Torfason, A. Manolescu, Dynamic Electrical Behavior of Halide Perovskite Based Solar Cells, Sol. Energy Mater. Sol. Cells 159 (2017) 197-203.

[12] Y. Zou, R.J. Holmes, Temperature-Dependent Bias Poling and Hysteresis in Planar Organo-Metal Halide Perovskite Photovoltaic Cells, Adv. Energy Mater. 6 (2016) 1501994.

[13] S. Ravishankar, O. Almora, C. Echeverría-Arrondo, E. Ghahremanirad, C. Aranda, A. Guerrero, F. Fabregat-Santiago, A. Zaban, G. Garcia-Belmonte, J. Bisquert, Surface Polarization Model for the Dynamic Hysteresis of Perovskite Solar Cells, J. Phys. Chem. Lett. 8 (2017) 915-921.

[14] H. Shen, D.A. Jacobs, Y. Wu, T. Duong, J. Peng, X. Wen, X. Fu, S.K. Karuturi, T.P. White, K. Weber, K.R. Catchpole, Inverted Hysteresis in $\mathrm{CH}_{3} \mathrm{NH}_{3} \mathrm{PbI}_{3}$ Solar Cells: Role of Stoichiometry and Band Alignment, J. Phys. Chem. Lett. 8 (2017) 2672-2680. 
[15] G. Richardson, S.E.J. O'Kane, R.G. Niemann, T.A. Peltola, J.M. Foster, P.J. Cameron, A.B. Walker, Can Slow-Moving Ions Explain Hysteresis in the CurrentVoltage Curves of Perovskite Solar Cells?, Energy Environ. Sci. 9 (2016) 1476-1485.

[16] R.A. Belisle, W.H. Nguyen, A.R. Bowring, P. Calado, X. Li, S.J.C. Irvine, M.D. McGehee, P.R.F. Barnes, B.C. O'Regan, Interpretation of Inverted Photocurrent Transients in Organic Lead Halide Perovskite Solar Cells: Proof of the Field Screening by Mobile Ions and Determination of the Space Charge layer Widths, Energy Environ. Sci. 10 (2017) 192-204.

[17] O. Almora, C. Aranda, I. Zarazua, A. Guerrero, G. Garcia-Belmonte, Noncapacitive Hysteresis in Perovskite Solar Cells at Room Temperature, ACS Energy Lett. 1 (2016) 209-215.

[18] O. Almora, K.T. Cho, S. Aghazada, I. Zimmermann, G.J. Matt, C.J. Brabec, M.K. Nazeeruddin, G. Garcia-Belmonte, Discerning Recombination Mechanisms and Ideality Factors through Impedance Analysis of High-Efficiency Perovskite Solar Cells, Nano Energy 48 (2018) 63-72.

[19] K.T. Cho, G. Grancini, Y. Lee, E. Oveisi, J. Ryu, O. Almora, M. Tschumi, P.A. Schouwink, G. Seo, S. Heo, J. Park, J. Jang, S. Paek, G. Garcia-Belmonte, M.K. Nazeeruddin, Selective Growth of Layered Perovskites for Stable and Efficient Photovoltaics, Energy Environ. Sci. 11 (2018) 952-959.

[20] G. Grancini, C. Roldán-Carmona, I. Zimmermann, E. Mosconi, X. Lee, D. Martineau, S. Narbey, F. Oswald, F. De Angelis, M. Graetzel, M.K. Nazeeruddin, OneYear Stable Perovskite Solar Cells by 2D/3D Interface Engineering, Nat. Commun. 8 (2017) 15684.

[21] Y. Rong, Y. Hu, S. Ravishankar, H. Liu, X. Hou, Y. Sheng, A. Mei, Q. Wang, D. Li, M. Xu, J. Bisquert, H. Han, Tunable Hysteresis Effect for Perovskite Solar Cells, Energy Environ. Sci. 10 (2017) 2383-2391.

[22] K.T.B. Jarvist M. Frost, Federico Brivio, Christopher H. Hendon, Mark van Schilfgaarde, and Aron Walsh, Atomistic Origins of High-Performance in Hybrid Halide Perovskite Solar Cells, Nano Lett. 14 (2014) 2584-2590.

[23] M. Sendner, P.K. Nayak, D.A. Egger, S. Beck, C. Muller, B. Epding, W. Kowalsky, L. Kronik, H.J. Snaith, A. Pucci, R. Lovrincic, Optical Phonons in 
Methylammonium Lead Halide Perovskites and Implications for Charge Transport, Mater. Horiz. 3 (2016) 613-620.

[24] N. Onoda-Yamamuro, T. Matsuo, H. Suga, Dielectric study of $\mathrm{CH}_{3} \mathrm{NH}_{3} \mathrm{PbX}_{3}(\mathrm{X}=$ Cl, Br, I), J. Phys. Chem. Solids 53 (1992) 935-939.

[25] O. Almora, A. Guerrero, G. Garcia-Belmonte, Ionic Charging by Local Imbalance at Interfaces in Hybrid Lead Halide Perovskites, Appl. Phys. Lett. 108 (2016) 043903.

[26] P. Lopez-Varo, J.A. Jiménez-Tejada, M. García-Rosell, J.A. Anta, S. Ravishankar, A. Bou, J. Bisquert, Effects of Ion Distributions on Charge Collection in Perovskite Solar Cells, ACS Energy Lett. 2 (2017) 1450-1453.

[27] M. García-Rosell, A. Bou, J.A. Jiménez-Tejada, J. Bisquert, P. Lopez-Varo, Analysis of the Influence of Selective Contact Heterojunctions on the Performance of Perovskite Solar Cells, J. Phys. Chem. C 122 (2018) 13920-13925.

[28] P. Calado, A.M. Telford, D. Bryant, X. Li, J. Nelson, B.C. O'Regan, P.R.F. Barnes, Evidence for Ion Migration in Hybrid Perovskite Solar Cells with Minimal Hysteresis, Nat. Commun. 7 (2016) 13831.

[29] S. van Reenen, M. Kemerink, H.J. Snaith, Modeling Anomalous Hysteresis in Perovskite Solar Cells, J. Phys. Chem. Lett. 6 (2015) 3808-3814.

[30] Y. Huang, S. Aharon, A. Rolland, L. Pedesseau, O. Durand, L. Etgar, J. Even, Influence of Schottky contact on the $\mathrm{C}-\mathrm{V}$ and $\mathrm{J}-\mathrm{V}$ characteristics of HTM-free perovskite solar cells, EPJ Photovolt. 8 (2017) 85501.

[31] K. Majhi, L. Bertoluzzi, D.A. Keller, H.-N. Barad, A. Ginsburg, A.Y. Anderson, R. Vidal, P. Lopez-Varo, I. Mora-Sero, J. Bisquert, A. Zaban, $\mathrm{Co}_{3} \mathrm{O}_{4}$ Based All-Oxide PV: A Numerical Simulation Analyzed Combinatorial Material Science Study, J. Phys. Chem. C 120 (2016) 9053-9060.

[32] C. Bat-El, W. Małgorzata, E. Lioz, High Efficiency and High Open Circuit Voltage in Quasi 2D Perovskite Based Solar Cells, Adv. Funct. Mater. 27 (2017) 1604733.

[33] E.A. Muljarov, S.G. Tikhodeev, N.A. Gippius, T. Ishihara, Excitons in SelfOrganized Semiconductor/Insulator Superlattices: PbI-Based Perovskite Compounds, Phys. Rev. B 51 (1995) 14370-14378.

[34] I.C. Smith, E.T. Hoke, D. Solis-Ibarra, M.D. McGehee, H.I. Karunadasa, A Layered Hybrid Perovskite Solar-Cell Absorber with Enhanced Moisture Stability, 
Angew. Chem. 126 (2014) 11414-11417.

[35] J.-W. Lee, Z. Dai, T.-H. Han, C. Choi, S.-Y. Chang, S.-J. Lee, N. De Marco, H. Zhao, P. Sun, Y. Huang, Y. Yang, 2D Perovskite Stabilized Phase-Pure Formamidinium Perovskite Solar Cells, Nat. Commun. 9 (2018) 3021.

[36] G.A. Nemnes, C. Besleaga, V. Stancu, D.E. Dogaru, L.N. Leonat, L. Pintilie, K. Torfason, M. Ilkov, A. Manolescu, I. Pintilie, Normal and Inverted Hysteresis in Perovskite Solar Cells, J. Phys. Chem. C 121 (2017) 11207-11214.

[37] W. Tress, J.P. Correa Baena, M. Saliba, A. Abate, M. Graetzel, Inverted CurrentVoltage Hysteresis in Mixed Perovskite Solar Cells: Polarization, Energy Barriers, and Defect Recombination, Adv. Energy Mater. 6 (2016) 1600396.

[38] O. Almora, C. Aranda, G. Garcia-Belmonte, Do Capacitance Measurements Reveal Light-Induced Bulk Dielectric Changes in Photovoltaic Perovskites?, J. Phys. Chem. C 122 (2018) 13450-13454.

[39] I. Zarazua, J. Bisquert, G. Garcia-Belmonte, Light-Induced Space-Charge Accumulation Zone as Photovoltaic Mechanism in Perovskite Solar Cells, J. Phys. Chem. Lett. 7 (2016) 525-528.

[40] M. Burgelman, P. Nollet, S. Degrave, Modelling Polycrystalline Semiconductor Solar Cells, Thin Solid Films 361-362 (2000) 527-532.

[41] A. Niemegeers, M. Burgelman, Effects of the Au/CdTe Back Contact on IV and CV Characteristics of Au/CdTe/CdS/TCO Solar Cells, J. Appl. Phys. 81 (1997) 28812886. 


\section{Supporting Information:}

\section{Ionic Dipolar Switching Hinders Charge Collection in Perovskite Solar Cells with Normal and Inverted Hysteresis}

Osbel Almora ${ }^{\mathrm{a}, \mathrm{b}, \mathrm{c}, *}$, Pilar Lopez-Varo ${ }^{\mathrm{d}}$, Kyung Taek Cho ${ }^{\mathrm{e}}$, Sadig Aghazada ${ }^{\mathrm{e}}$, Wei Meng $^{\mathrm{a}}$, Yi Hou ${ }^{\mathrm{a}}$, Carlos Echeverría-Arrondo ${ }^{\mathrm{b}}$, Iwan Zimmermann ${ }^{\mathrm{e}}$, Gebhard J. Matt ${ }^{\mathrm{a}}$, Juan A. Jiménez-Tejada ${ }^{\mathrm{d}}$, Christoph J. Brabec ${ }^{\mathrm{a}}$, Mohammad Khaja Nazeeruddin ${ }^{\mathrm{e}}$, Germà Garcia-Belmonte ${ }^{\mathrm{b}, *}$

${ }^{a}$ Institute of Materials for Electronics and Energy Technology (i-MEET), FriedrichAlexander Universität Erlangen-Nürnberg, 91058 Erlangen, Germany

${ }^{\mathrm{b}}$ Institute of Advanced Materials (INAM), Universitat Jaume I, 12006 Castelló, Spain

${ }^{\mathrm{c}}$ Erlangen Graduate School in Advanced Optical Technologies (SAOT), FriedrichAlexander Universität Erlangen-Nürnberg, 91052 Erlangen, Germany

d Departamento de Electrónica y Tecnología de Computadores CITIC-UGR, Universidad de Granada, 18071 Granada, Spain

e Group for Molecular Engineering of Functional Materials, Institute of Chemical Sciences and Engineering, École Polytechnique Fédérale de Lausanne, CH-1951 Sion, Switzerland

* corresponding authors

E-mail addresses: osbel.almora@fau.de (O. Almora), garciag@uji.es (G. GarciaBelmonte)

Keywords: perovskite solar cells, ionic polarization, hysteresis, photocurrent 


\section{S1. Introduction}
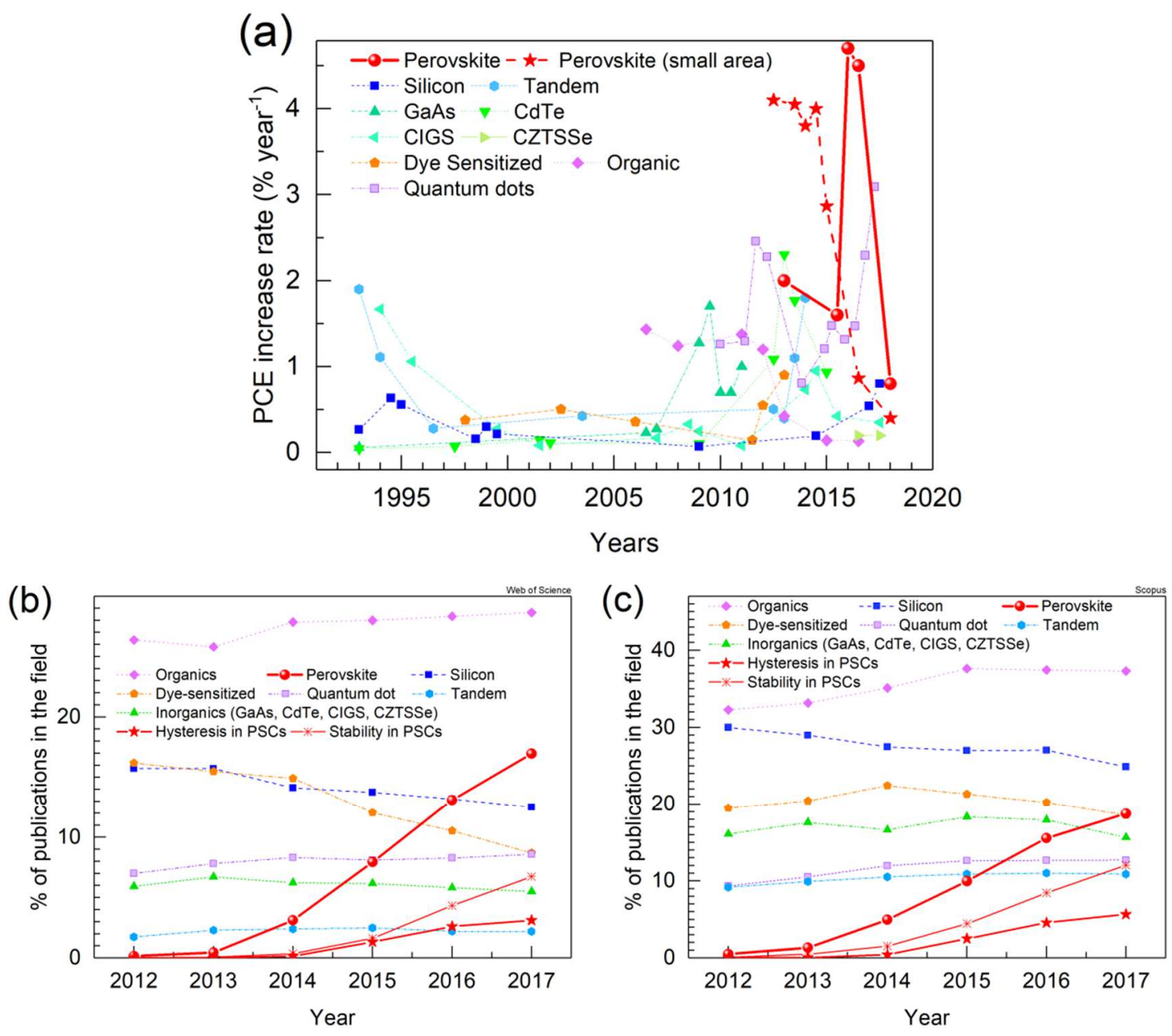

Fig. S6. (a) Power conversion efficiency (PCE) increase rate of different solar cells technologies over the last 25 years and percentages of publications by technologies in the field of solar cells/photovoltaics from (b) Web of Science and (c) Scopus since 2012. PCE data taken from M.A. Green et al. "Solar cell efficiency tables" [1] and NREL efficiency chart. Web of Science/Scopus publications data accessed on 07/09/2018. 


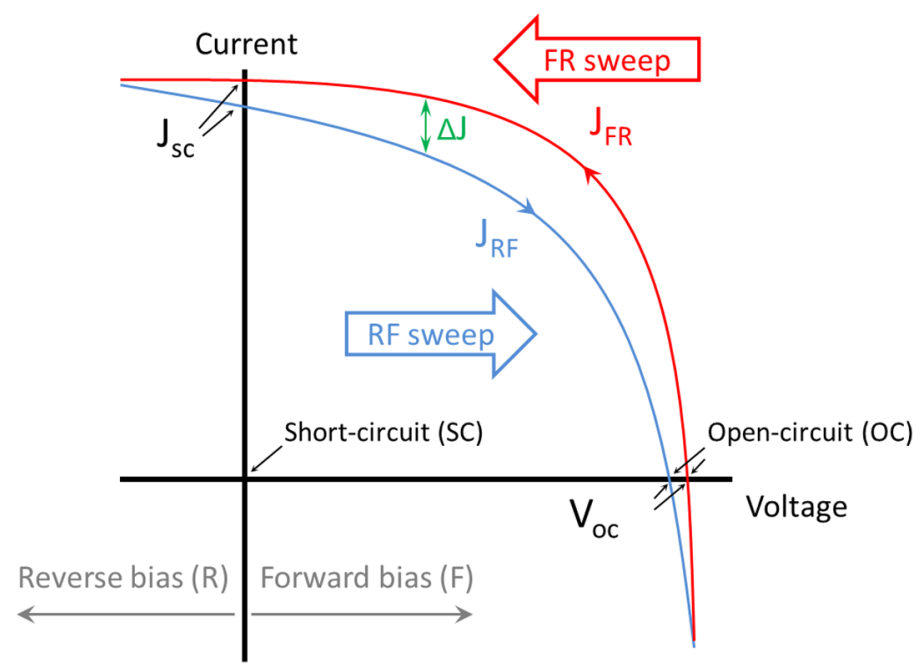

Fig. S7. Sketch of normal hysteresis (NHyst) in current-voltage curves of PSCs and notations used in this work. Importantly, note that forward/reverse bias is not forward/reverse sweep direction, as indistinctly used in the notation from some authors. Here we save the terms forward/reverse for the applied voltage ranges, as usually taken in semiconductor device physics. Forward/backward or Forwards/reverse sweep directions are discarded in favor of RF/FR, respectively.
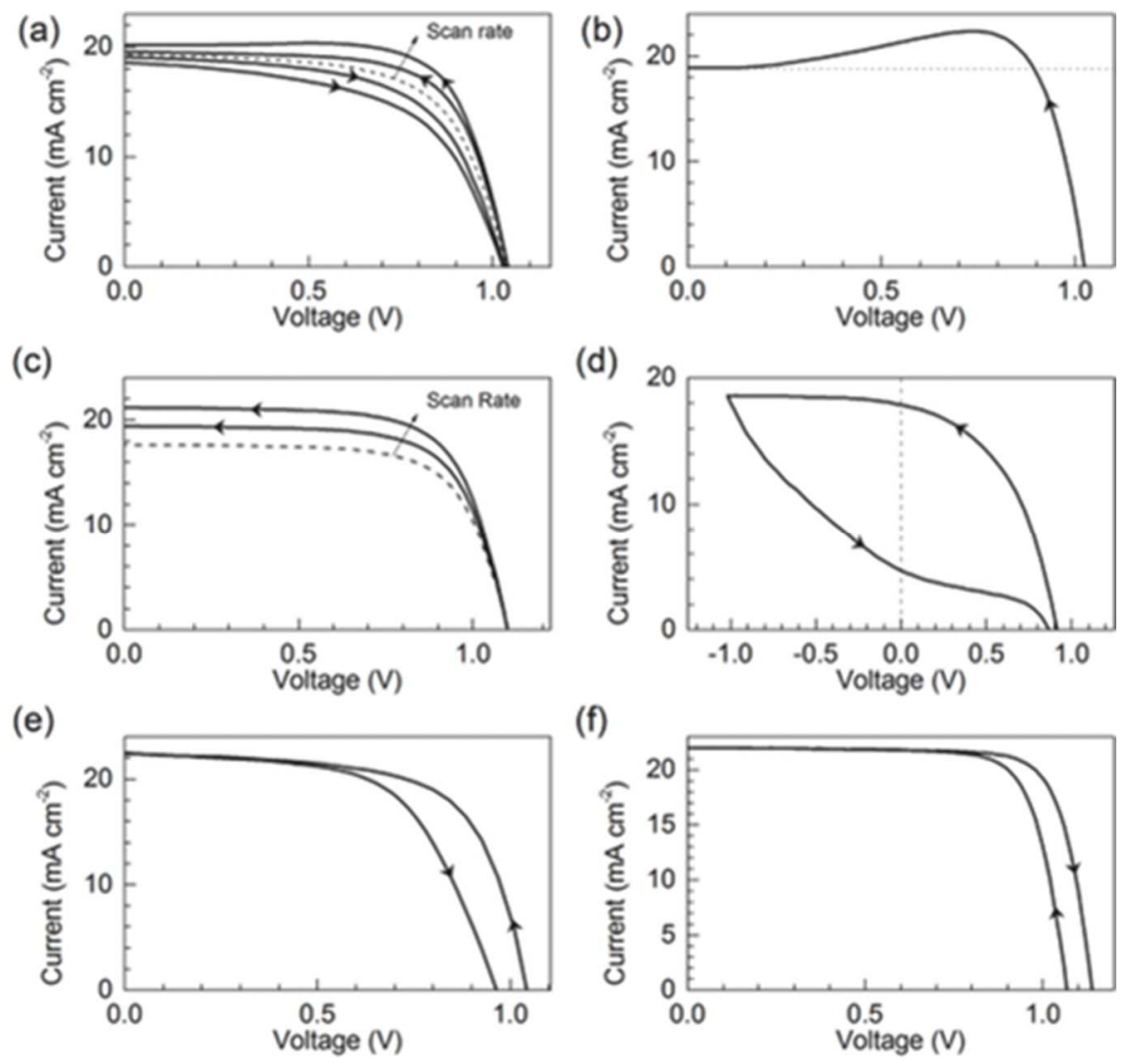
Fig. S8. Summary of dynamic hysteresis trends in PSCs. The arrows show the direction of the sweep. (a) Capacitive hysteresis, (b) "bump" close to $V_{o c}$, (c) apparent photocurrent enhancement in FR direction, (d) strong decay of $J_{s c}$ in RF direction, (e) apparent $V_{o c}$ shift for RF scan, (f) inverted hysteresis. (a,c) Dashed lines indicate the steady-state curve, and (b,d) $J_{\mathrm{sc}}$ and zero bias, respectively. Reproduced with permission.[2] Copyright 2018, John Wiley \& Sons, Inc. Originally adapted with permission from ref. [3] Copyright 2017, American Chemical Society.

(a)

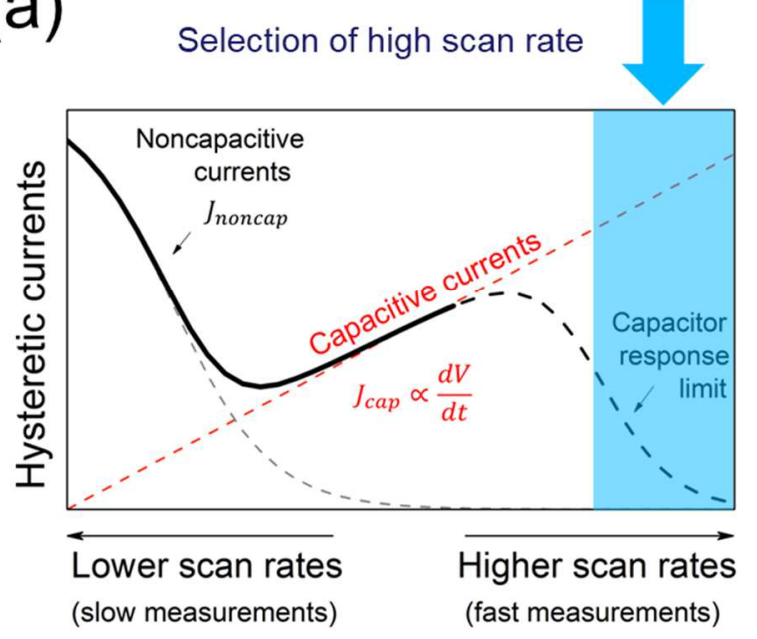

(b)

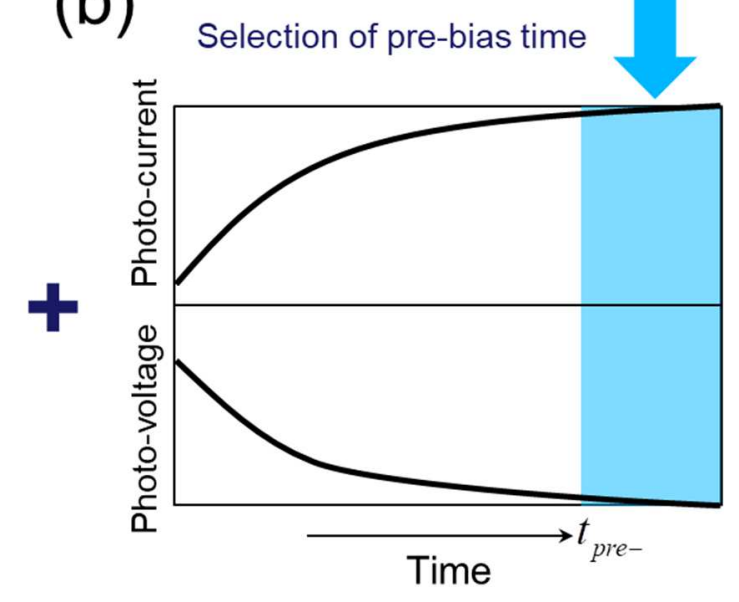

Fig. S9. Sketch of assumptions taken in this work in order to design the experiment. Adapted with permission from ref. [4] Copyright 2016, American Chemical Society.

\section{S2. Experimental}

\section{S2.1. Fabrication of MAPI-based devices}

Conductive FTO glass (NSG10) was sequentially cleaned by sonication in a $2 \%$ Helmanex solution and isopropanol for $15 \mathrm{~min}$ each. A 20-50 $\mathrm{nm}$ titania blocking layer was applied on the substrates by spraying a solution of titanium diisopropoxide bis(acetylacetonate) in ethanol at $450{ }^{\circ} \mathrm{C}$. For the $200-300 \mathrm{~nm}$ mesoporous $\mathrm{TiO}_{2}$ layer, 30 NR-D titania paste from Dyesol diluted in ethanol $(150 \mathrm{mg} / \mathrm{ml})$ was applied by spincoating at $4000 \mathrm{rpm}$ for 10 s followed by a sintering step at $500{ }^{\circ} \mathrm{C}$ for $20 \mathrm{~min}$. After cooling down the substrates a Li-treatment was applied by spin-coating $60 \mu \mathrm{l}$ of a solution of Tris(bis(trifluoromethylsulfonyl)imide) (Li-TFSI) in acetonitrile $(10 \mathrm{mg} / \mathrm{ml})$ 
onto the mesoporous layer, followed by a sintering step at $500{ }^{\circ} \mathrm{C}$ for $10 \mathrm{~min}$ to decompose the Li-salt as previously described [5].

The 3D MAPI perovskite layers were fabricated by a single step spin-coating procedure reported by Seok et al. [6] (Fig.1a). For the perovskite precursor solution, $484 \mathrm{mg}$ of $\mathrm{PbI}_{2}$ (TCI) and $159 \mathrm{mg}$ methylammonium iodide (Dyesol) were dissolved in $800 \mu \mathrm{l}$ dimethyl sulfoxide (DMSO). For the 2D perovskite on the titania side $2 \mathrm{~mol} \%$ of 5 Ammonium valeric acid (5-AVAI) was added to the perovskite precursor solution. The perovskite solution was spun at $4000 \mathrm{rpm}$ for $30 \mathrm{~s}$ using a ramp of $2000 \mathrm{rpms}^{-1}$. Ten seconds prior to the end of the spin-coating sequence $100 \mu \mathrm{l}$ chlorobenzene were poured onto the spinning substrate. Afterwards, the substrates were transferred onto a heating plate and annealed at $100{ }^{\circ} \mathrm{C}$ for $45 \mathrm{~min}$. In order to form a thin layer of $2 \mathrm{D}$ perovskite on top of the perovskite capping layer, the samples were cooled down and a solution of benzimidazolium iodide (BI) in isopropanol $(5 \mathrm{mg} / \mathrm{ml})$ was dynamically spin-coated onto the perovskite layers at $3000 \mathrm{rpm}$. The samples were afterwards annealed for additional 10 minutes at $100{ }^{\circ} \mathrm{C}$.

The hole-transporting material, spiro-OMeTAD (Merk) was applied from a $70 \mathrm{mM}$ solution in chlorobenzene. Tert-butylpyridine (Tbp), Tris(2-(1H-pyrazol-1-yl)-4-tertbutylpyridine)cobalt(III) (FK209) and Li-TFSI were added as additives. $330 \mathrm{~mol} \% \mathrm{Tbp}$, $50 \mathrm{~mol} \% \mathrm{Li}$-TFSI from a 1.8M stock solution in acetonitrile and 3 mol\% FK209 from a $0.25 \mathrm{M}$ stock solution in acetonitrile was mixed to the spiro-OMeTAD solution. The final HTM solution was spin-coated dynamically onto the perovskite layers at 4000rpm for $20 \mathrm{~s}$. The gold electrodes were deposited by thermal evaporation of $80 \mathrm{~nm}$ gold using a shadow mask under high vacuum conditions.

\section{S2.2. Fabrication of $\mathrm{Cs}_{0.1} \mathrm{FA}_{0.74} \mathrm{MA}_{0.13} \mathrm{PbI}_{2.48} \mathrm{Br}_{0.39}$-based devices}

FTO glass substrates (NSG) were sequentially cleaned with the hellmanex solution, acetone, and ethanol. Thus, a compact $\mathrm{TiO}_{2}$ layer was coated on the FTO substrate heated by spray pyrolysis deposition at $450^{\circ} \mathrm{C}$. A precursor solution was a diluted titanium diisopropoxide (Sigma-Aldrich) in ethanol $(0.6 \mathrm{ml}: 10 \mathrm{ml})$. After cooling, mesoporous $\mathrm{TiO}_{2}$ film ( 200 nm) was prepared coating a diluted $\mathrm{TiO}_{2}$ paste (Dyesol 30 
NR-D) solution in ethanol. Right after spin-coating, the substrates were sintered on a hot plate at $500^{\circ} \mathrm{C}$ for $20 \mathrm{~min}$. For doping $\mathrm{Li}$ on $\mathrm{TiO}_{2}$, films were treated with $0.1 \mathrm{M}$ Lithium bistrifluoromethanesulfonimidate solution (Li-TFSI, Aldrich) in acetonitrile by spin-coating at $5000 \mathrm{rpm}$ for $10 \mathrm{~s}$, and sintered again at $500^{\circ} \mathrm{C}$ for $30 \mathrm{~min}$.

Subsequently, a 3D perovskite solution (Fig. 1c) was prepared by mixing FAI (1.1 M), $\mathrm{PbI}_{2}(1.15 \mathrm{M}), \mathrm{MABr}(0.2 \mathrm{M})$ and $\mathrm{PbBr}_{2}(0.2 \mathrm{M})$ in a mixed solvent (DMF: DMSO = 4: 1 volume ratio). Another solution of $\mathrm{CsPbI}_{3}$ was also prepared as $1.15 \mathrm{M}$ in DMF: DMSO (same volume ratio). And then, $\left(\mathrm{FAPbI}_{3}\right)_{0.85}\left(\mathrm{MAPbBr}_{3}\right)_{0.15}$ and $\mathrm{CsPbI}_{3}$ solutions were mixed as $10 \mathrm{vol} \%$ ratio. The solution was then spin-coated at $2000 \mathrm{rpm}$ for $10 \mathrm{~s}$ and continuously at $5000 \mathrm{rpm}$ for $30 \mathrm{~s}$ in nitrogen glove box. After entering the second step, $100 \mu \mathrm{L}$ of anhydrous trifluorotoluene was poured at 15 seconds before the finish. Films were then annealed at $100^{\circ} \mathrm{C}$ for $60 \mathrm{~min}$. For forming additional 2D perovskite film on top of this perovskite film, and between $\mathrm{TiO}_{2}$ and CFMPIB, substrates were treated with a PEAI isopropanol solution. $100 \mu \mathrm{L}$ of PEAI solution $(15 \mathrm{mg} / \mathrm{ml})$ were spin-coated on the $\mathrm{TiO}_{2}$ substrate or the as-prepared perovskite films at $4000 \mathrm{rpm}$, and annealed at $100^{\circ} \mathrm{C}$ for $5 \mathrm{~min}$.

Finally, Spiro-OMeTAD was spin-coated at $4000 \mathrm{rpm}$ for $20 \mathrm{~s}$. The $70 \mathrm{mM}$ SpiroOMeTAD solution was prepared by dissolving in chlorobenzene with 4-tertbutylpyridine, Li-TFSI in acetonitrile, and $\mathrm{Co}[\mathrm{t}-\mathrm{BuPyPz}]_{3}[\mathrm{TFSI}]_{3}$ (FK209) in acetonitrile at the molar ratio of Spiro:FK209:Li-TFSI:TBP of 1:0.03:0.5:3.3. Devices were completed with a thermal evaporation of $70 \mathrm{~nm}$ thickness gold rear electrode.

\section{S2.3. Fabrication of $F A_{0.83} M A_{0.17} P_{1.1} B_{0.22} I_{2.98}$-based devices}

The patterned ITO substrates were ultrasonically cleaned using acetone and isopropanol for 10 minutes each. For "n-i-p" device, the $\mathrm{SnO}_{2}$ layer was spin coated at 3000 r.p.m. for $30 \mathrm{~s}$ on cleaned ITO substrate and annealed the film at $150{ }^{\circ} \mathrm{C}$ for $15 \mathrm{~min} .10 \mathrm{mg} / \mathrm{ml}$ 
PC60BM solution in chlorobenzene was deposited on to the $\mathrm{SnO}_{2}$ at a speed of 3000 r.p.m. The as-prepared perovskite precursor solution $\left(\mathrm{FA}_{0.83} \mathrm{MA}_{0.17} \mathrm{~Pb} 1.1 \mathrm{Br}_{0.22} \mathrm{I}_{2.98}\right)$ was filtered using a $0.45 \mu \mathrm{m}$ PTFE syringe filter and coated onto substrates at a speed of 4000 r.p.m for 20 s. The substrate was quickly quenched by $0.3 \mathrm{ml}$ chlorobenzene and further annealed on a hot plate at $110{ }^{\circ} \mathrm{C}$ for 30 seconds (low crystallinity film) and 10 minutes (high crystallinity film), respectively. The prepared PDCBT polymer HTMs were spin-coated at 2000 r.p.m for 15 s. Subsequently, Ta- $\mathrm{WO}_{\mathrm{x}}$ was coated onto PDCBT at a speed of 2000 r.p.m for 10 s. Finally, a 100-nm-thick Au counter electrode was deposited by thermal evaporation.

\section{S2.4. Performance of the studied devices}

(a)

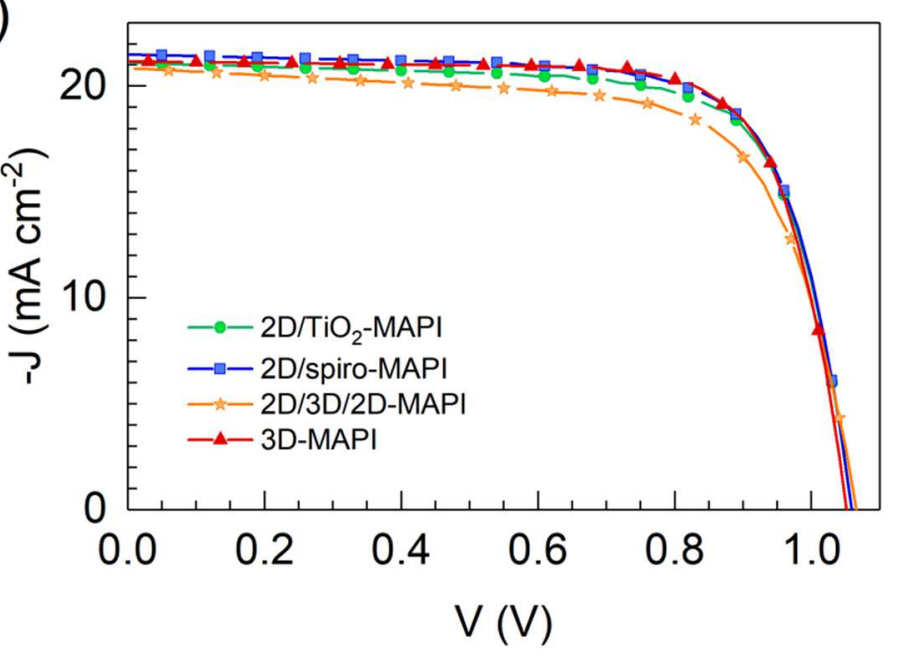



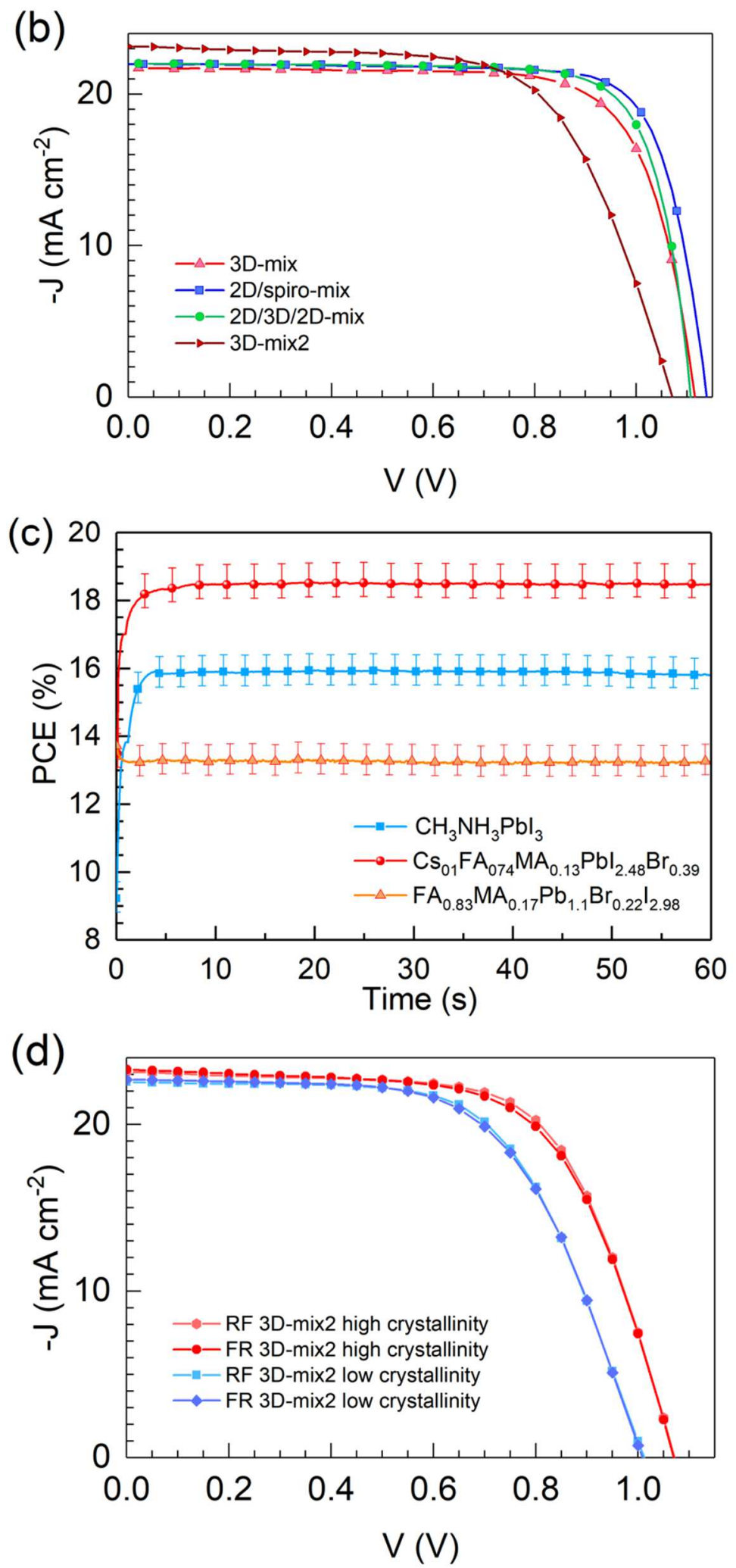

Fig. S10. $J-V$ curves of samples before (fresh) and after (stressed) characterization 
sequence for structures including (a) MAPI and (b) $\mathrm{Cs}_{0.1} \mathrm{FA}_{0.74} \mathrm{MA}_{0.13} \mathrm{PbI}_{2.48} \mathrm{Br}_{0.39}$ and $\mathrm{FA}_{0.83} \mathrm{MA}_{0.17} \mathrm{~Pb}_{1.1} \mathrm{Br}_{0.22} \mathrm{I}_{2.98}$ perovskites, as indicated (see labeling Fig. 1). The illumination was $100 \mathrm{mWcm}^{-2} \mathrm{AM} 1.5 \mathrm{G}$ and the bias was swept in FR direction at 50 $\mathrm{mV} \cdot \mathrm{s}^{-1}$ at room conditions. In (c), the evolution of maxim power output is presented for representative devices, and the error bars indicate the average difference between the PCE from $J-V$ curves and that at steady maxim power voltage. In (d), the $J-V$ curves of two $\mathrm{FA}_{0.83} \mathrm{MA}_{0.17} \mathrm{~Pb}_{1.1} \mathrm{Br}_{0.22} \mathrm{I}_{2.98}$ devices with different degrees of crystallinity illustrate nearly hysteresis-free behavior at $300 \mathrm{mV} \cdot \mathrm{s}^{-1}$. (a-c) Adapted with permission from ref. [7] Copyright 2018, Elsevier Ltd.

Table S1. Performance parameters from Fig. S10, corresponding to the $J-V$ curves of the samples before the characterization sequence. See Fig. 1a,c for structure labeling and ref. [7] for more details.

\begin{tabular}{|l|c|c|c|c|}
\hline \multicolumn{1}{|c|}{ Device } & $\begin{array}{c}V_{c} \\
(\mathrm{mV})\end{array}$ & $\begin{array}{c}J_{s c} \\
\left(\mathrm{~mA} \mathrm{~cm}^{-2}\right)\end{array}$ & FF $(\%)$ & PCE $(\%)$ \\
\hline 2D/TiO2-MAPI & 1059 & 21.1 & 73.2 & 16.4 \\
\hline 2D/spiro-MAPI & 1060 & 21.5 & 73.2 & 16.7 \\
\hline 2D/3D/2D-MAPI & 1066 & 20.8 & 69.1 & 15.3 \\
\hline 3D-MAPI & 1052 & 21.2 & 74.9 & 16.7 \\
\hline 2D/spiro-mix & 1138 & 22.0 & 78.3 & 19.6 \\
\hline 2D/3D/2D-mix & 1107 & 22.0 & 78.2 & 19.1 \\
\hline 3D-mix & 1115 & 21.7 & 74.8 & 18.1 \\
\hline 3D-mix2 & 1070 & 23.1 & 65.5 & 16.2 \\
\hline
\end{tabular}




\section{S3. Results and discussion}
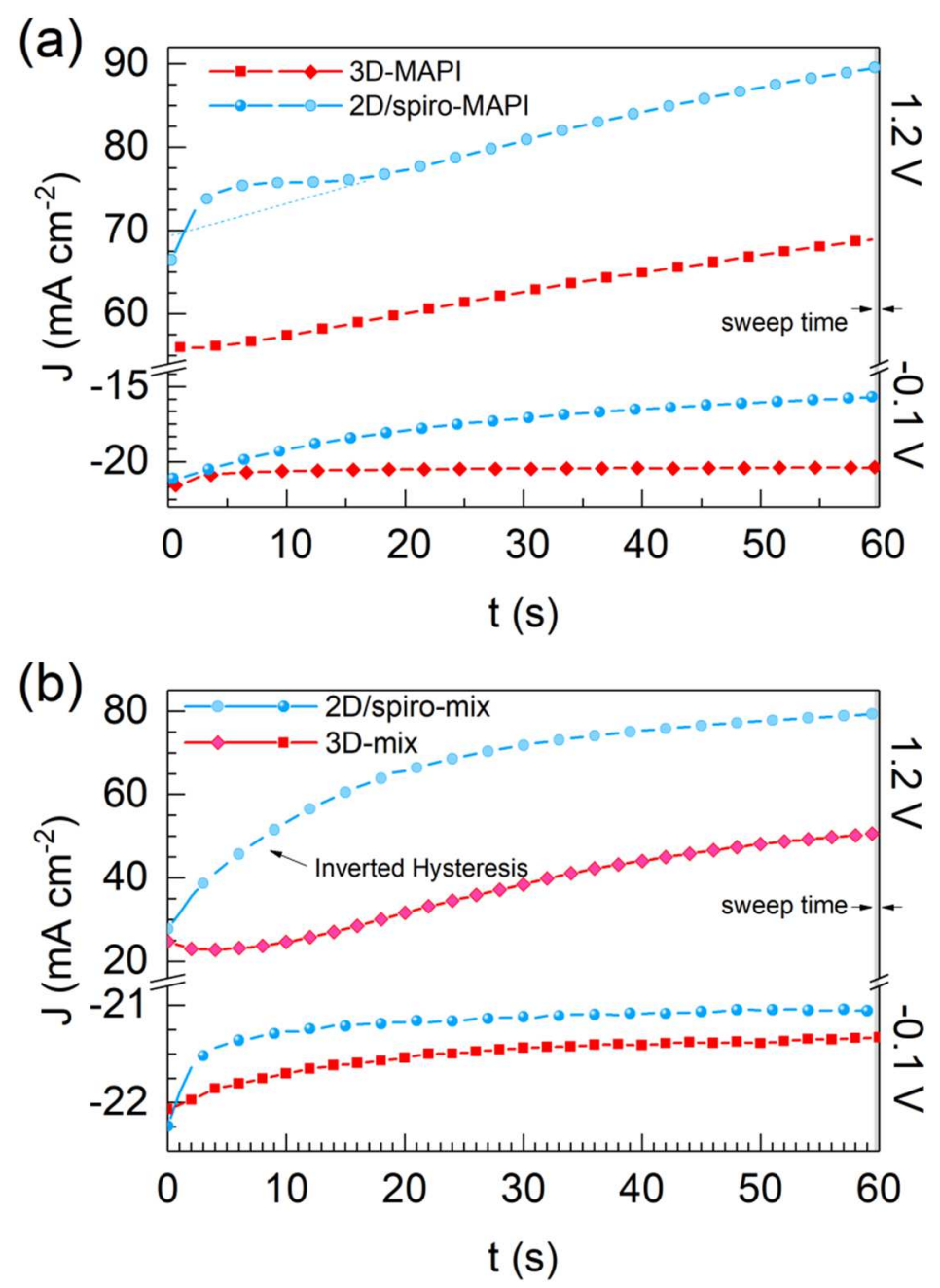


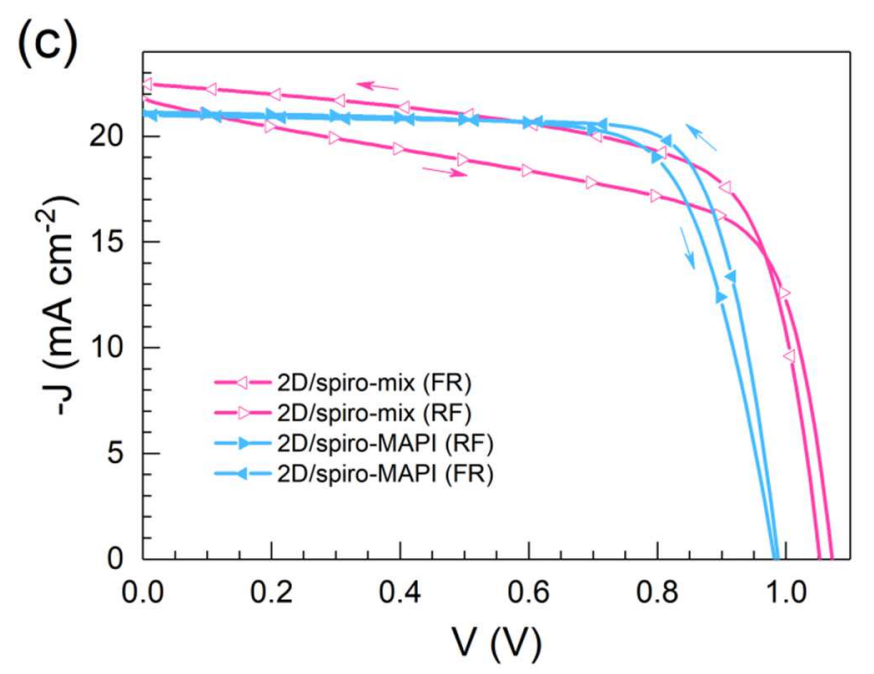

Fig. S11. Current-time evolution during pre-bias for representative (a) MAPI- and (b) CFMPIBbased samples, as indicated. Stabilization of photo-current (bottom of the figures) is reached after a minute. In general, the change in photo-current is always larger for samples including 2D capping. During forward bias pooling (two top figures) the warming-up of samples seems to increase current indistinctly for all samples. (c) $J$ - $V$ curves examples of top hysteretic devices, as indicated, without pre-bias, at $2 \mathrm{~V} \mathrm{~s}^{-1}$ and with continuous sweep $(-0.1 \mathrm{~V} ; 1.2 \mathrm{~V} ;-0.1 \mathrm{~V})$. In general in absence of large pre-bias and slower scan rates the patterns of Fig. 1b,d are not reproducible.

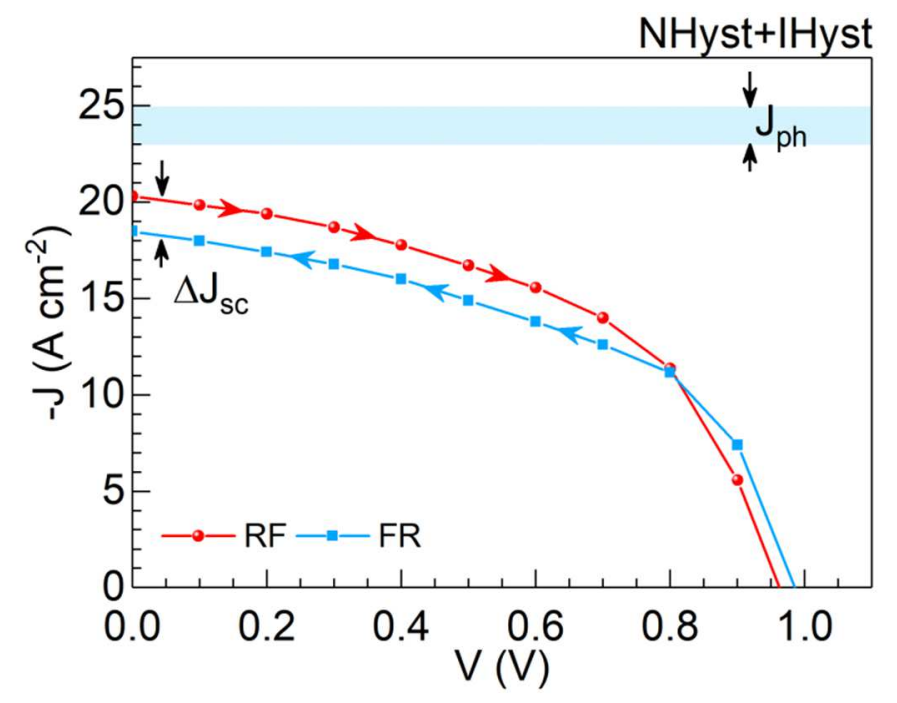

Fig. S12. Simulated J-V curves including NHyst and IHyst. 


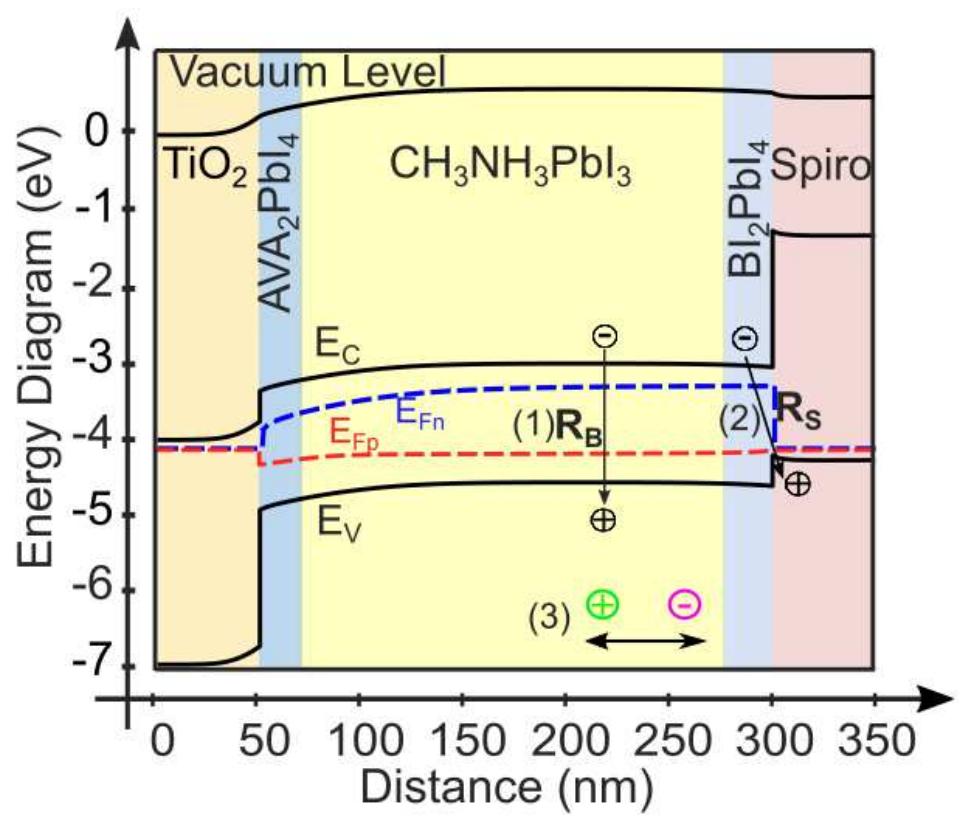

Fig. S13. Energy diagram of a MAPI-based PSCs including 2D layers working in short-circuit ( $\left.V_{a p p}=0 \mathrm{~V}\right)$ and under illumination. $\mathrm{E}_{\mathrm{Fn}}$ and $\mathrm{E}_{\mathrm{Fp}}$ are the quasi-Fermi levels for electron and holes, respectively, and $\mathrm{E}_{\mathrm{C}}$ and $\mathrm{E}_{\mathrm{V}}$ are the conduction and valence band levels, respectively. Physical mechanisms that can help to interpret the effect of the 2D layers: (i) a discontinuity in the conduction band energy, $\Delta \mathrm{E}_{\mathrm{C}}$, at the ETL/perovskite interface, and a discontinuity in the valence band energy, $\Delta \mathrm{E}_{\mathrm{v}}$, at the $\mathrm{HTL} /$ perovskite interface; (ii) surface recombination rate $R_{\mathrm{S}}$ between electrons and holes accumulated at the interfaces; (iii) bulk recombination rate $R_{\mathrm{B}}$ in the perovskite; and (iv) migration of ions.

Table S2. Summary of parameters used for simulations of Fig. 3, Fig. 4 and Fig. S7-S11. See further details on equations in our previous works $[2,8,9]$.

\begin{tabular}{|c|c|c|c|c|}
\hline Parameter, symbol (units) & ETL n-type & \multicolumn{2}{|c|}{ Perovskite } & HTL p-type \\
\hline & & NHyst & IHyst & \\
\hline Layer length, $L(\mathrm{~nm})$ & 50 & \multicolumn{2}{|r|}{200} & 50 \\
\hline Relative permittivity, $\varepsilon_{r}$ & 20 & 26 & 10 & 3 \\
\hline Electron-hole mobility, $\mu\left(\mathrm{cm}^{2} \mathrm{~V}^{-1} \mathrm{~s}^{-1}\right)$ & 1 & 10 & 5 & $10^{-2}$ \\
\hline Band-gap, $E_{g}(\mathrm{eV})$ & 3.2 & \multicolumn{2}{|r|}{1.6} & 3 \\
\hline Acceptor concentration, $N_{A}\left(\mathrm{~cm}^{-3}\right)$ & 0 & \multirow{2}{*}{\multicolumn{2}{|c|}{$\frac{10^{17}}{0}$}} & $10^{17}$ \\
\hline Donor concentration, $N_{D}\left(\mathrm{~cm}^{-3}\right)$ & $10^{18}$ & & & 0 \\
\hline Mobile ions concentration, $N_{\text {ion }}\left(\mathrm{cm}^{-3}\right)$ & 0 & \multicolumn{2}{|c|}{\begin{tabular}{c|c}
\multicolumn{1}{c}{0} \\
& $8 \times 10^{18}(\mathrm{SC})$ \\
$8 \times 10^{18}$ & $4.5 \times 10^{18}(\mathrm{OC})$
\end{tabular}} & 0 \\
\hline Generation of e-h pair rate, $G\left(\mathrm{~cm}^{-3} \mathrm{~s}^{-1}\right)$ & 0 & \multicolumn{2}{|c|}{$7.5 \times 10^{21}$} & 0 \\
\hline $\begin{array}{c}\text { Bulk recombination coefficient, } \beta_{B} \\
\left(\mathrm{~cm}^{3} \mathrm{~s}^{-1}\right)\end{array}$ & $10^{-8}$ & \multicolumn{2}{|r|}{$10^{-8}$} & $10^{-8}$ \\
\hline
\end{tabular}




\begin{tabular}{|c|c|c|c|}
\hline $\begin{array}{c}\text { Surface recombination coefficient, } \beta_{S} \\
\left(\mathrm{~cm}^{3} \mathrm{~s}^{-1}\right)\end{array}$ & $\begin{array}{c}0 ; \\
10^{-12} \text { (Fig. 3c) }\end{array}$ & 0 & $\begin{array}{c}0 ; \\
10^{-12} \text { (Fig. 3c) }\end{array}$ \\
\hline
\end{tabular}
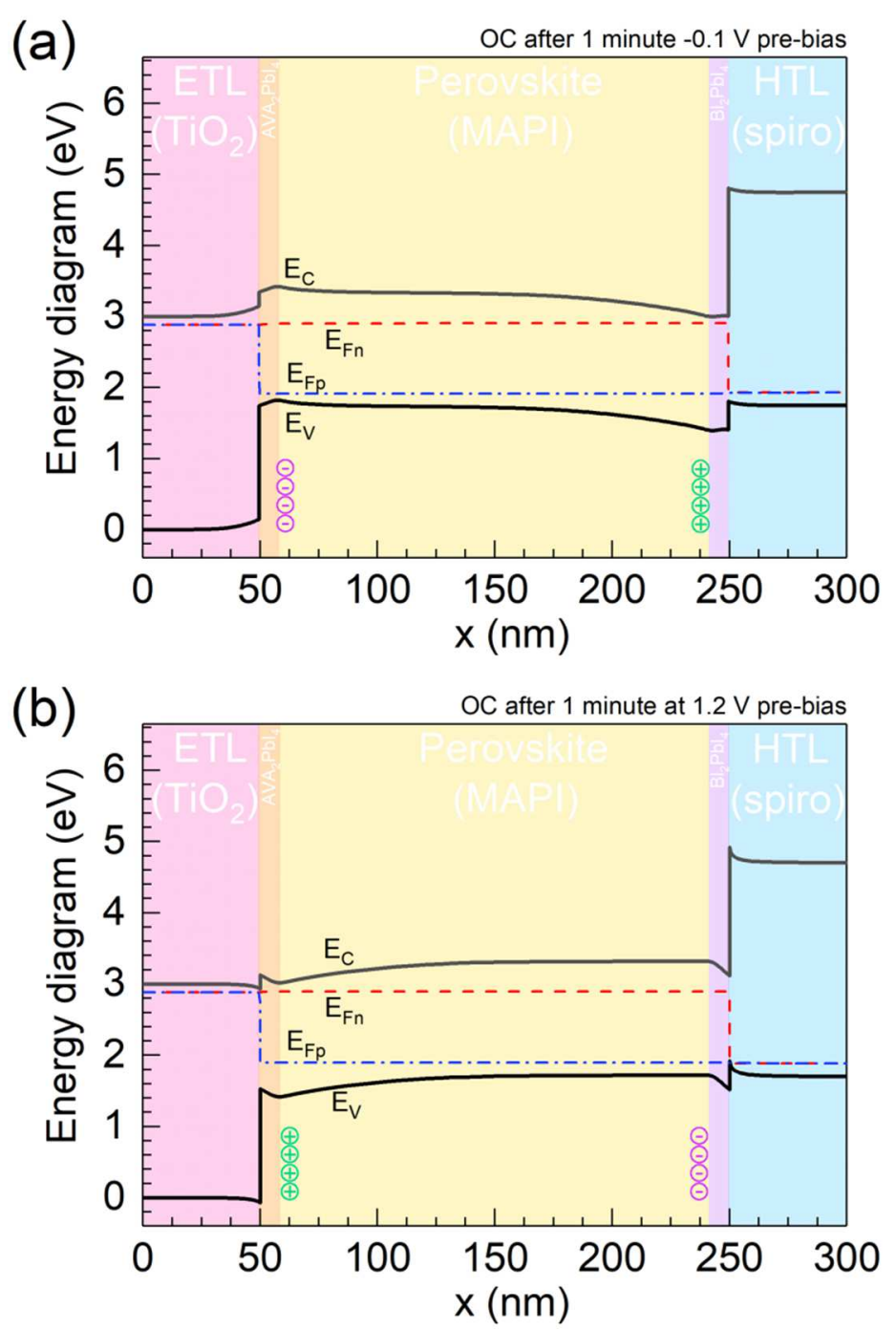


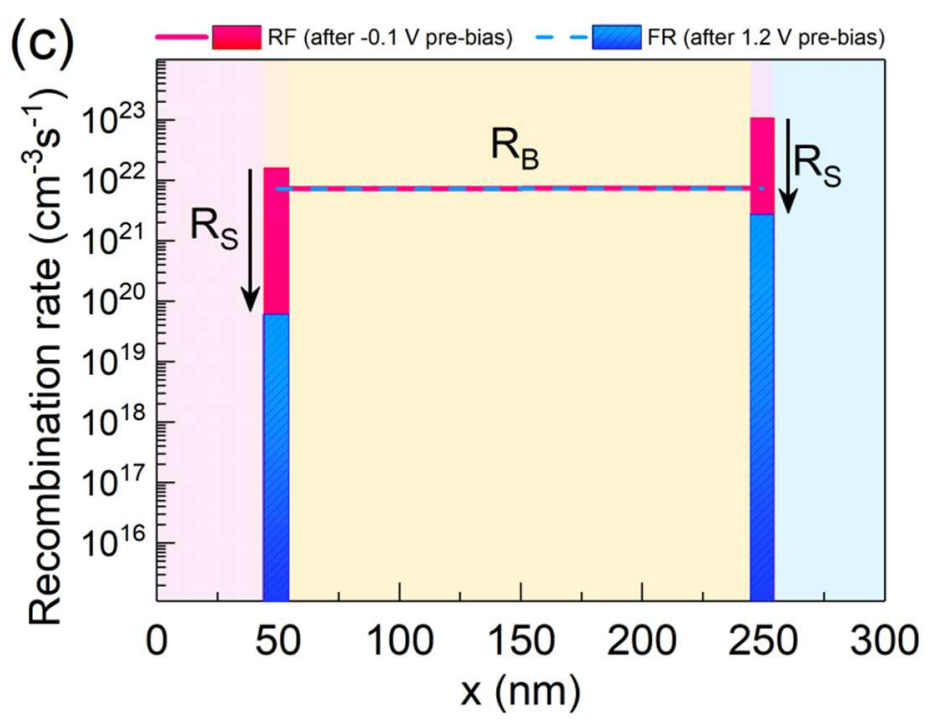

Fig. S14. Energy diagrams of PSC including 2D layers working under illumination at opencircuit $\left(J=0 \mathrm{~A} \cdot \mathrm{m}^{-2}\right)$. (a) Calculations made at point "B" in the RF scan of Fig. 3a. (b) Calculations made at point "C" in the FR scan of Fig. 3a. (c) Magnitude of the surface recombination $R_{\mathrm{S}}$ and bulk recombination $R_{\mathrm{B}}$ for cases (a) and (b). $E_{F n}$ and $E_{F p}$ are the quasiFermi levels of electrons and holes, respectively, and $E_{C}$ and $E_{V}$ are the conduction and valence band levels, respectively.
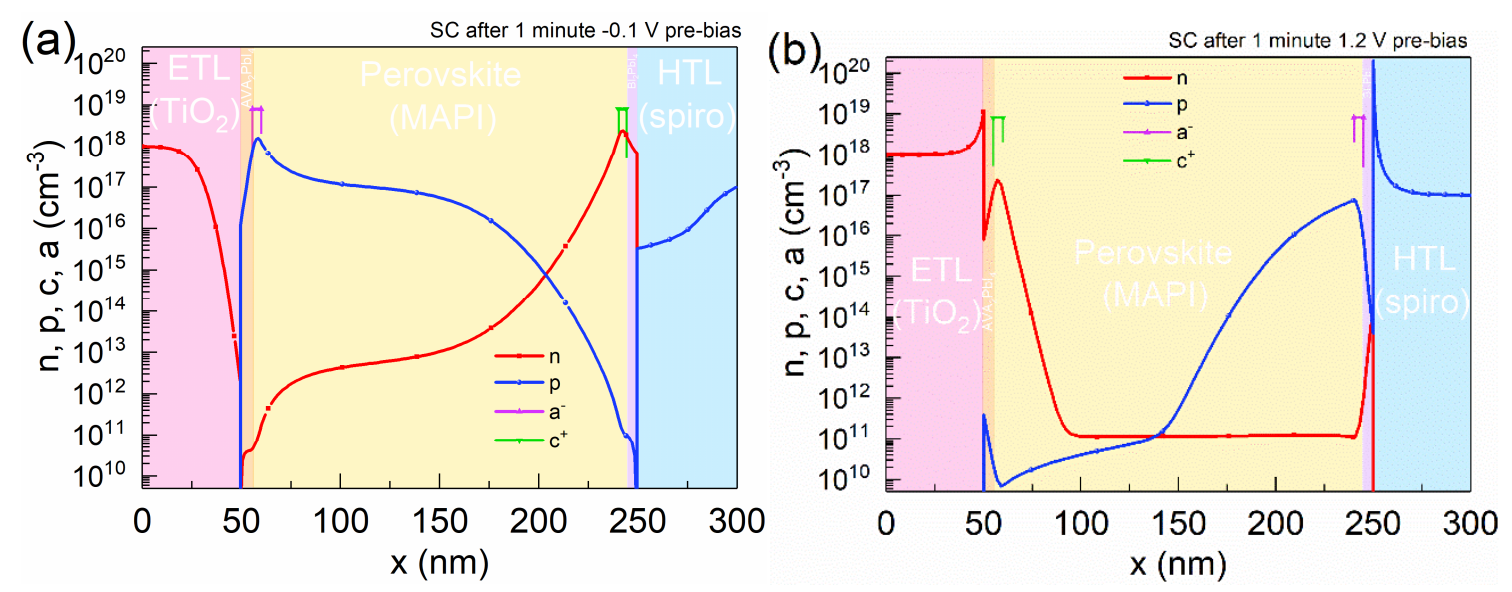

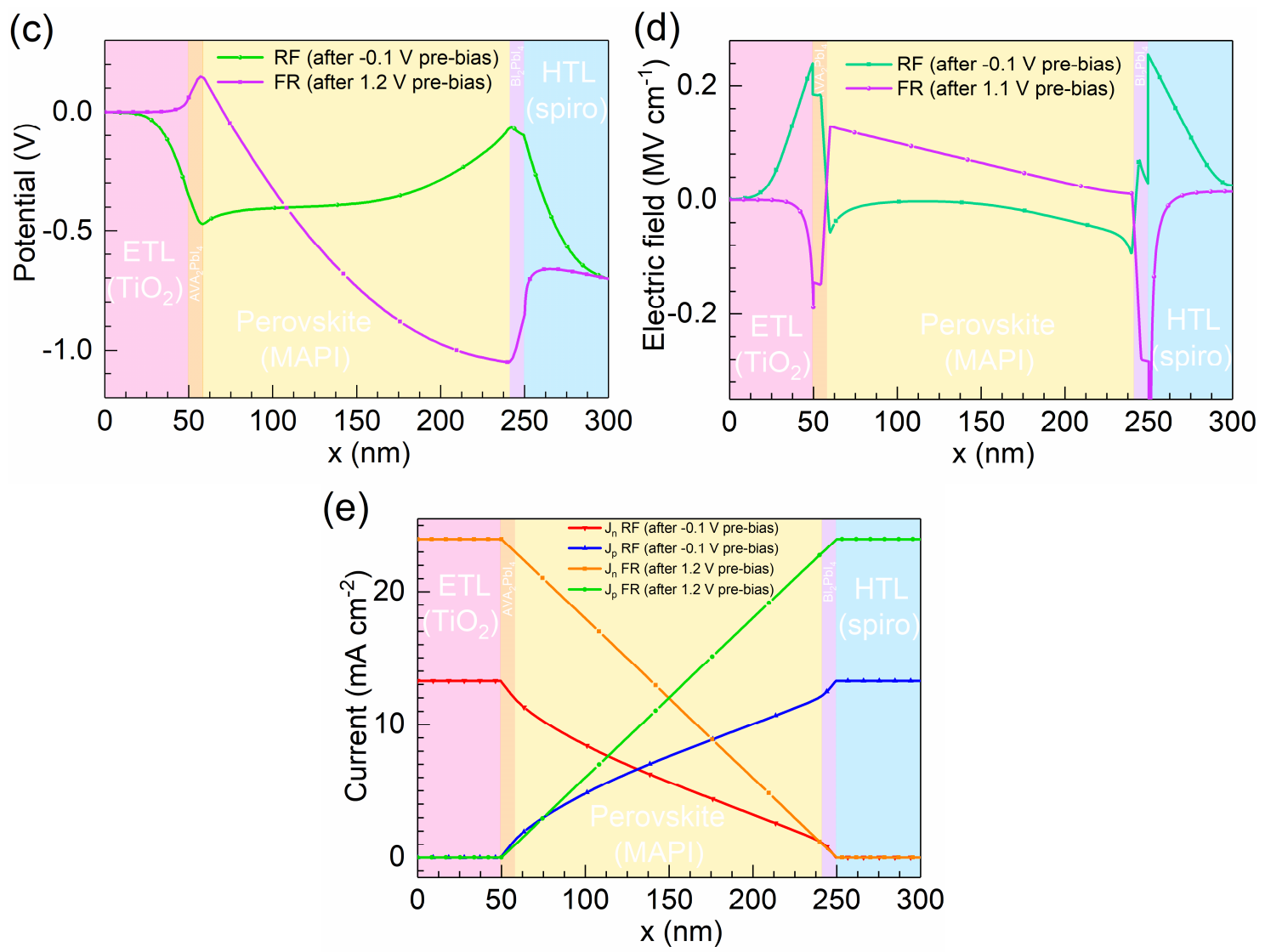

Fig. S15. Distribution of the electrical variables along the MAPI-cells analyzed in Fig. 3 at short-circuit. Distributions of free charge carriers (electrons $n$ and holes $p$ ) and ions (anions a and cations $\mathrm{c}^{+}$) along the structure calculated at (a) point (C) in the RF scan of Fig. 3a, (b) point (B) in the FR scan of Fig. 3a. Similarly, resultant distributions of (c) potential, (d) electric field and (e) current density, calculated at points (C) and (B) of Fig. 3a. See Table S2 for simulation parameters. 

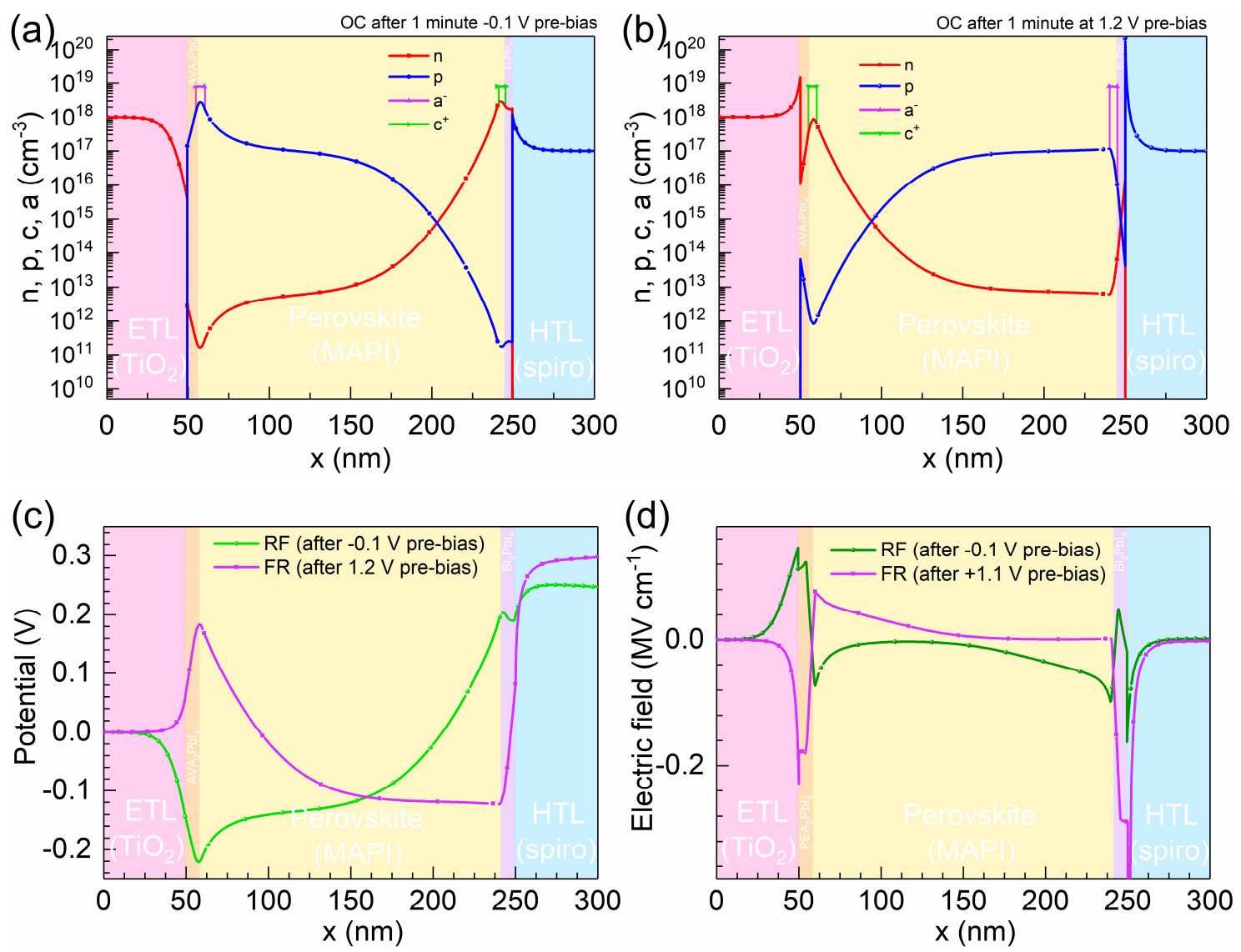

Fig. S16. Distribution of electrical variables along the MAPI-cells analyzed in Fig. S14 at opencircuit. Distributions of free charge density (electrons, $n$, and holes, $\mathrm{p}$ ) and ions (anions $\mathrm{a}^{-}$and cations $\mathrm{c}^{+}$) along the structure calculated at (a) point (A) in the FR scan of Fig. 3a, (b) point (C) in the RF scan of Fig. 3a. Similarly, resultant distributions of (c) potential, and (d) electric field. See Table S2 for simulation parameters. 
Table S3. Fitting parameters used for modeling curves in Fig. S12 using Eq. 3.

\begin{tabular}{|c|c|c|c|c|c|c|c|}
\hline Structure & $\begin{array}{c}\Delta J_{s c} \\
\left(\mathrm{~mA} \mathrm{~cm}^{-2}\right)\end{array}$ & $\begin{array}{c}J_{\alpha} \\
\left(\mathrm{mA} \mathrm{cm}^{-2}\right)\end{array}$ & $\begin{array}{c}J_{\beta} \\
\left(\mathrm{mA} \mathrm{cm}^{-2}\right)\end{array}$ & $\begin{array}{c}V_{\alpha} \\
(\mathrm{mV})\end{array}$ & $\begin{array}{c}V_{\beta} \\
(\mathrm{mV})\end{array}$ & $n_{\alpha}$ & $n_{\beta}$ \\
\hline 3D-MAPI & 0 & 10.8 & 30.4 & 635 & 1070 & 4.7 & 2.6 \\
\hline 2D/TiO $-M A P I$ & 1.5 & 12.4 & 62.8 & 325 & 1065 & 5.5 & 2.8 \\
\hline 2D/3D/2D-MAPI & 5.1 & 13.0 & 62.8 & 476 & 1082 & 7.9 & 4.0 \\
\hline 2D/spiro-MAPI & 6.0 & 10.7 & 62.8 & 335 & 1045 & 7.3 & 3.0 \\
\hline 3D-mix & -2.2 & 2.4 & 27.4 & 365 & 1006 & 9.5 & 2.6 \\
\hline 2D/spiro-mix & -7.2 & 36.8 & 32.4 & 1120 & 1002 & 0.8 & 2.0 \\
\hline 2D/3D/2D-mix & -0.1 & 16.3 & 8.2 & 857 & 1068 & 4.8 & 1.3 \\
\hline 2D-mix2 & -0.2 & 9 & 44.7 & 687 & 815 & 2.6 & 6.1 \\
\hline 2D-mix2 (low crystallinity) & -0.3 & 10.2 & 23.0 & 465 & 919 & 1.5 & 6.9 \\
\hline
\end{tabular}

\section{S3.1. Analyzing the empirical $\Delta J$ and the 2-diode model.}

Attending to each term and parameter in Eq. 3, we propose that the nearly voltageindependent mechanism of $\Delta J_{s c}$ can respond to the switching and/or creation of the ionic dipoles, which are favored by the presence of the 2D interlayers in MAPI devices. The reason is that the $2 \mathrm{D}$ perovskites are known to limit the ionic mobility [10], thus other morphology factors (e.g. crystallinity) with the same effect (altering ionic mobility) can favor or hinder the occurrence of $\Delta J_{s c}$.

On the two voltage-dependent $\alpha / \beta$ terms in Eq. 3, which mostly affects the FF in the $J-V$ curves, three issues can be highlighted. First, they may account for hysteretic processes in (i) bulk MAPI and/or (ii) throughout pin holes since they are irrespective on the presence of $2 \mathrm{D}$ capping layers. Second, the presence of two $\alpha / \beta$ opposing currents can be understood due to reordered charge profiles as a consequence of (i) the pre- 
biasing and/or (ii) the voltage scan rate direction in a form of displacement currents. Third, the $J_{\alpha} \alpha(V)$ and $J_{\beta} \beta(V)$ currents concern to (i) different ionic species and/or (ii) different material region than those related with $\Delta J_{s c}$. These observations are summarized in Table S4.

Accordingly, on the interpretation of $J_{\alpha} \alpha(V)$ and $J_{\beta} \beta(V)$ terms in Eq. 3 we can argue two general natures: faradaic and non-faradaic currents. On the side of faradaic currents the finiteness of $J_{\alpha}$ and $J_{\beta}$ and the general shape of the terms (current steps around activation voltages $V_{\alpha}$ and $V_{\beta}$ ) resembles the Nerst-Monod model [11], as found in our previous work for slow scan rates $J-V$ curve sweeps [4], However the occurrence of reversible redox reactions at fast scan rates, as in our experiment, is quite unlikely.

On the other hand, as non-faradaic currents, the "S" shape of $J_{\alpha} \alpha(V)$ in Eq. 3 and Fig. S12 resembles the $J-V$ curve of polycrystalline CdTe solar cells $[12,13]$, where the high injection current of a main junction diode is limited by an opposing Schottky diode. Consequently, we propose that $\mathrm{p}^{+} \mathrm{np}$ or $\mathrm{n}^{+} \mathrm{pn}$ local charge density profiles (baseoverloaded transistors or switchable consecutive ionic dipoles) are oriented during prebias and/or voltage scans in sort of channels which produce NHyst or IHyst, depending on the polarization. These ionic related charge profiles can behave similarly to the dipolar sheets assumed in the drift-diffusion simulations: in quasi-steady-state during the fast scan. In this scenario, the current modulation may follow the transcendent equation 


$$
J_{\text {2diodes }}(V)=J_{s 1}\left(\exp \left[\frac{q V_{1}}{m_{1} k_{B} T}\right]-1\right)-J_{p h 12}=J_{s 2}\left(\exp \left[-\frac{q\left(V-V_{1}\right)}{m_{2} k_{B} T}\right]-1\right)
$$

where $V_{1}$ and $V-V_{1}=V_{2}$ are the voltage drops at each diode/dipole. The meanings of $J_{s 1,2}$ and $m_{1,2}$ can be respectively associated with $J_{s}$ and $m$ per each diode, but care must be taken when interpreting numerical fittings since both parameters complement each other when scaling the exponentials. Similarly, $J_{p h 12}$ accounts for an absolute generated current, whose sign is that of the predominant absorbing diode.

The numerical solution of Eq. (S4) matches well the $J_{\alpha} \alpha(V)$ behavior with the parameters of Table S5, as shown with solid lines in Fig. S12c,d. An important effect of this interpretation is that the hysteretic features increase with illumination, which is a wide known experimental behavior [2]. Subsequently, also the $J_{\beta} \beta(V)$ term can be associated and similarly fitted to $J_{2 \text { diodes }}$ (see Fig. S12 and Table S5). However, despite the presence of a second diode in this configuration could be true, the large values of $J_{\beta}$ and $V_{\beta}$ (see Eq. 3 and Table S3) approximate $\beta(V) \approx \exp \left[q\left(V-V_{\beta}\right) / n_{\beta} k_{B} T\right]$, which make sense as a main diode voltage-shifted by the $V_{\beta}$ drop due to series resistances or the second diode. This could be also a pin hole effect.

Table S4. Summary on analysis of parameters of Eq. 3

\begin{tabular}{|l|c|c|}
\hline Analysis points & $\Delta J_{s c}$ & $J_{\alpha} \alpha(V)$ and $J_{\beta} \beta(V)$ \\
\hline Key-related layer & 2D capping & 3D bulk absorber and/or pin holes \\
\hline Dependence on pre-biasing & Yes & $\begin{array}{c}\text { Yes, but possibly related with other } \\
\text { factors, e.g. scan direction }\end{array}$ \\
\hline
\end{tabular}




\begin{tabular}{|l|c|c|}
\hline Voltage-dependency & Moderate or none towards SC & Yes \\
\hline $\begin{array}{l}\text { Main affected PCE } \\
\text { parameter }\end{array}$ & $J_{s c}$ & $F F$ and $V_{o c}$ \\
\hline Related type of hysteresis & $\begin{array}{c}\text { NHyst for MAPI; } \\
\text { IHyst for CFMPIB }\end{array}$ & Nhyst for $J_{\alpha} \alpha(V) ;$ \\
IHyst for $J_{\beta} \beta(V)$
\end{tabular}

Table S5. Fitting parameters used for matching numerical $J_{\text {2diode }}(V)$ function to empirical analytical currents $J_{\alpha} \alpha(V)$ and $J_{\beta} \beta(V)$ curves in Fig. S12

\begin{tabular}{|c|c|c|c|c|c|c|c|c|c|c|}
\hline \multirow{2}{*}{ Structure } & \multicolumn{9}{|c|}{$J_{\alpha} \alpha(V)$} & \multicolumn{7}{c|}{$-J_{\beta} \beta(V)$} \\
& \multirow{2}{*}{$\begin{array}{c}J_{p h 12} \\
\left(\mathrm{~mA} \mathrm{~cm}^{-2}\right)\end{array}$} & $\begin{array}{c}J_{s 1} \\
\left(\mathrm{~mA} \mathrm{~cm}^{-2}\right)\end{array}$ & $\begin{array}{c}J_{s 2} \\
\left(\mathrm{~mA} \mathrm{~cm}^{-2}\right)\end{array}$ & $m_{1}$ & $m_{2}$ & $\begin{array}{c}J_{p h 12} \\
\left(\mathrm{~mA} \mathrm{~cm}^{-2}\right)\end{array}$ & $\begin{array}{c}J_{s 1} \\
\left(\mathrm{~mA} \mathrm{~cm}^{-2}\right)\end{array}$ & $J_{s 2}$ \\
$\left(\mathrm{~mA} \mathrm{~cm}^{-2}\right)$ & $m_{1}$ & $m_{2}$ \\
\hline 3D-MAPI & 7.0 & 0.02 & 3.8 & 4.7 & 4.7 & 0 & 31 & $1.1 \times 10^{-5}$ & 2.6 & 2.8 \\
\hline 2D/TiO 2 -MAPI & 8.2 & 0.5 & 4.2 & 5.6 & 5.5 & 0 & 62 & $2.5 \times 10^{-5}$ & 2.6 & 2.8 \\
\hline 2D/3D/2D-MAPI & 7.5 & 0.6 & 5.4 & 7.9 & 7.9 & 0 & 62 & $1.8 \times 10^{-3}$ & 4.0 & 4.0 \\
\hline 2D/spiro-MAPI & 7 & 0.7 & 3.7 & 7.2 & 7.4 & 0 & 62 & $9.0 \times 10^{-5}$ & 3.0 & 3.0 \\
\hline 3D-mix & 1.4 & 0.3 & 1.0 & 9.9 & 9.9 & 0 & 27 & $9.1 \times 10^{-6}$ & 2.6 & 2.6 \\
\hline 2D/spiro-mix & 0 & $10^{-14}$ & 10 & 1.3 & 1.0 & 0 & 32 & $1.3 \times 10^{-7}$ & 2.0 & 2.0 \\
\hline 2D/3D/2D-mix & 5.7 & 0.011 & 10.5 & 4.8 & 4.8 & 0 & 8.3 & $2 \times 10^{-10}$ & 1.3 & 1.7 \\
\hline 3D-mix2 & -3.5 & 0.017 & 5.5 & 4.7 & 1.8 & 0 & 44.7 & 0.03 & 7.0 & 4.0 \\
\hline 3D-mix2 & 0 & 0.025 & 10.2 & 3.6 & 1.2 & 0.12 & 21.7 & 0.084 & 6.1 & 6.3 \\
(low crystallinity) & & & & & & & & & & \\
\hline
\end{tabular}
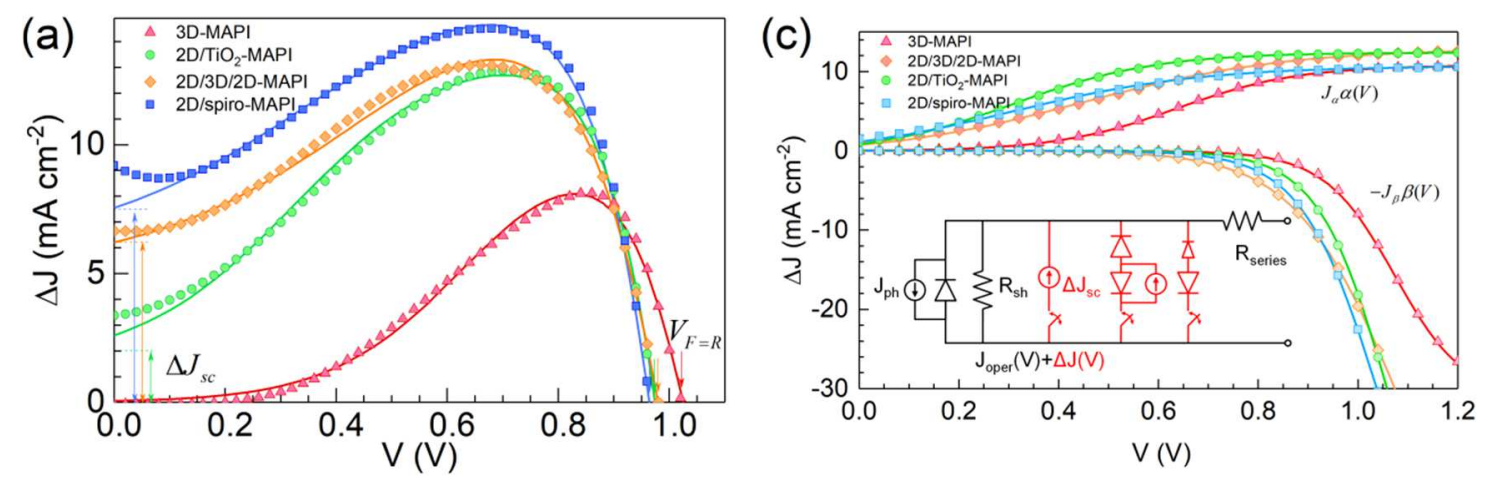

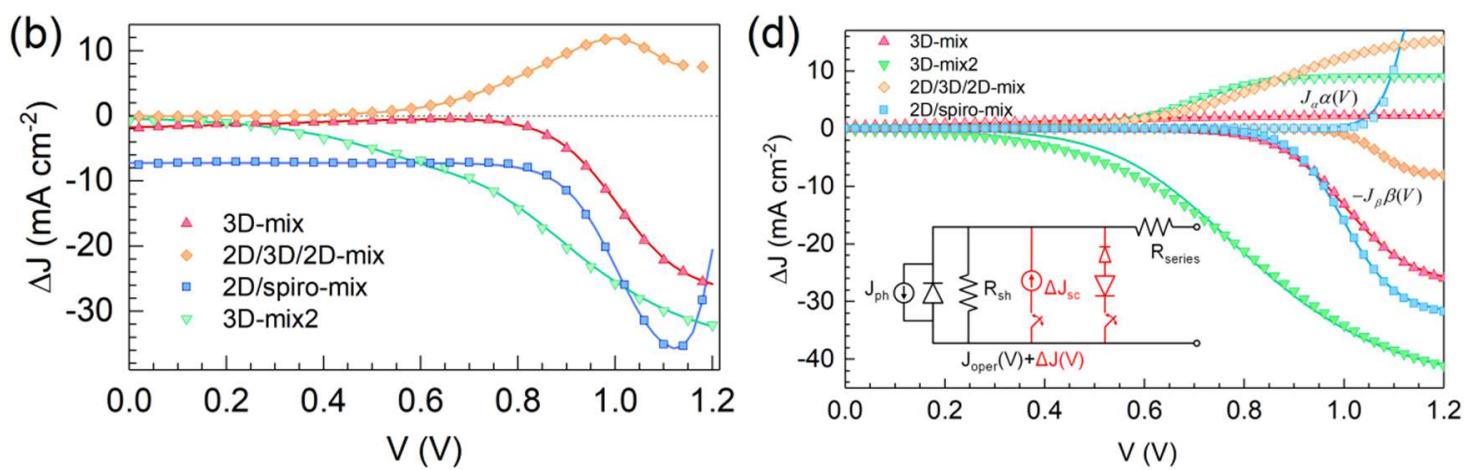

Fig. S17. (a, b) Experimental (dots) current difference between scan directions of $J-V$ curves of Fig. 1b,d and fittings (lines) to Eq. (3) whose fitting parameters are summarized in Table S3. (c, d) Analytical empirical components $J_{\alpha} \alpha(V)$ and $-J_{\beta} \beta(V)$ of Eq. (3) evaluated at the fitting parameters of Table S3 (dots) and corresponding fittings (solid lines) to the numerical twodiodes Eq. (S1) with parameters of Table S5 for MAPI- and CFMPIB-devices, as indicated. Inset the corresponding DC equivalent circuits.

\section{S4. References}

[1] M.A. Green, Y. Hishikawa, E.D. Dunlop, D.H. Levi, J. Hohl-Ebinger, A.W.Y. HoBaillie, Solar Cell Efficiency Tables (version 51), Prog. Photovoltaics 26 (2018) 3-12.

[2] P. Lopez-Varo, J.A. Jiménez-Tejada, M. García-Rosell, S. Ravishankar, G. GarciaBelmonte, J. Bisquert, O. Almora, Device Physics of Hybrid Perovskite Solar Cells: Theory and Experiment, Adv. Energy Mater. 8 (2018) 1702772.

[3] S. Ravishankar, O. Almora, C. Echeverría-Arrondo, E. Ghahremanirad, C. Aranda, A. Guerrero, F. Fabregat-Santiago, A. Zaban, G. Garcia-Belmonte, J. Bisquert, Surface Polarization Model for the Dynamic Hysteresis of Perovskite Solar Cells, J. Phys. Chem. Lett. 8 (2017) 915-921.

[4] O. Almora, C. Aranda, I. Zarazua, A. Guerrero, G. Garcia-Belmonte, Noncapacitive Hysteresis in Perovskite Solar Cells at Room Temperature, ACS Energy Lett. 1 (2016) 209-215.

[5] F. Giordano, A. Abate, J.P. Correa Baena, M. Saliba, T. Matsui, S.H. Im, S.M. Zakeeruddin, M.K. Nazeeruddin, A. Hagfeldt, M. Graetzel, Enhanced Electronic Properties in Mesoporous $\mathrm{TiO}_{2}$ via Lithium Doping for High-Efficiency Perovskite 
Solar Cells, Nat. Commun. 7 (2016) 10379.

[6] N.J. Jeon, J.H. Noh, Y.C. Kim, W.S. Yang, S. Ryu, S.I. Seok, Solvent Engineering for High-Performance Inorganic-Organic Hybrid Perovskite Solar Cells, Nat. Mater. 13 (2014) 897-903.

[7] O. Almora, K.T. Cho, S. Aghazada, I. Zimmermann, G.J. Matt, C.J. Brabec, M.K. Nazeeruddin, G. Garcia-Belmonte, Discerning Recombination Mechanisms and Ideality Factors through Impedance Analysis of High-Efficiency Perovskite Solar Cells, Nano Energy 48 (2018) 63-72.

[8] P. Lopez-Varo, J.A. Jiménez-Tejada, M. García-Rosell, J.A. Anta, S. Ravishankar, A. Bou, J. Bisquert, Effects of Ion Distributions on Charge Collection in Perovskite Solar Cells, ACS Energy Lett. 2 (2017) 1450-1453.

[9] M. García-Rosell, A. Bou, J.A. Jiménez-Tejada, J. Bisquert, P. Lopez-Varo, Analysis of the Influence of Selective Contact Heterojunctions on the Performance of Perovskite Solar Cells, J. Phys. Chem. C 122 (2018) 13920-13925.

[10] C. Bat-El, W. Małgorzata, E. Lioz, High Efficiency and High Open Circuit Voltage in Quasi 2D Perovskite Based Solar Cells, Adv. Funct. Mater. 27 (2017) 1604733.

[11] C.I. Torres, A.K. Marcus, P. Parameswaran, B.E. Rittmann, Kinetic Experiments for Evaluating the Nernst-Monod Model for Anode-Respiring Bacteria (ARB) in a Biofilm Anode, Environ. Sci. Technol. 42 (2008) 6593-6597.

[12] M. Burgelman, P. Nollet, S. Degrave, Modelling Polycrystalline Semiconductor Solar Cells, Thin Solid Films 361-362 (2000) 527-532.

[13] A. Niemegeers, M. Burgelman, Effects of the Au/CdTe Back Contact on IV and CV Characteristics of Au/CdTe/CdS/TCO Solar Cells, J. Appl. Phys. 81 (1997) 28812886. 